Network Working Group

Request for Comments: 3814

Category: Standards Track
T. Nadeau

Cisco Systems, Inc.

C. Srinivasan

Bloomberg L.P.

A. Viswanathan

Force10 Networks, Inc.

June 2004

Multiprotocol Label Switching (MPLS) Forwarding Equivalence

Class To Next Hop Label Forwarding Entry (FEC-To-NHLFE)

Management Information Base (MIB)

Status of this Memo

This document specifies an Internet standards track protocol for the Internet community, and requests discussion and suggestions for improvements. Please refer to the current edition of the "Internet Official Protocol Standards" (STD 1) for the standardization state and status of this protocol. Distribution of this memo is unlimited.

Copyright Notice

Copyright (C) The Internet Society (2004).

Abstract

This memo defines a portion of the Management Information Base (MIB) for use with network management protocols in the Internet community. In particular, it describes managed objects for defining, configuring, and monitoring Forwarding Equivalence Class (FEC) to Next Hop Label Forwarding Entry (NHLFE) mappings and corresponding actions for use with Multiprotocol Label switching (MPLS).

Table of Contents

1. Introduction . . . . . . . . . . . . . . . . . . . . . 2

2. Terminology. . . . . . . . . . . . . . . . . . . . . . . . 3

3. Conventions Used In This Document. . . . . . . . . . . . . . . 3

4. The Internet-Standard Management Framework . . . • • • . . . . 3

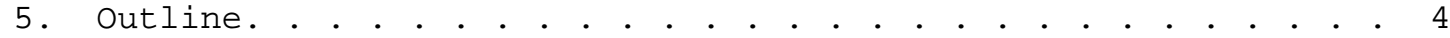

5.1. mplsftnTable . . . . . . . . . . . . . . . . . . . . . . . . 4 5.1.1. Advantages of Address Ranges Over CIDR Prefixes. 4

5.2. mplsfTNMaptable. . . . . . . . . . . . . . . . . . 5 5.2.1. Indexing Requirements. $\cdot$. . . . . . . . . . . 5 5.2.2. How the Current Indexing Works . . . . . . . . 5 5.3. mplsftnperftable . . . . . . . . . . . . . . . . . . . .7

6. Avoiding Retrieval-Modification Interactions . . . . . . . . 7 
7. Example Illustrating MIB Module Components . . . . . . . . . 8

7.1. Sample FTN Rules . . . . . . . . . . . . . . . . 8

7.2. Creating FTN Entries and Applying them to Interfaces . 9

7.3. Mapping an FTN Entry to Multiple Interfaces. . • • • . 10

7.4. Inserting an Entry Into Existing List. . . . . • • • • 11

7.5. Pictorial Tabular Relationship . . . . . . . . . . . 13

7.6. Deleting an Entry. . . . . . . . . . . . . . . . 14

8. The Use of RowPointer. • . • . • • • • • . . . . . . . . 16

9. MPLS-FTN-STD-MIB Definitions . . . . . . . . . . . . . . 16

10. Security Considerations. . . . . . . . . . . . . . . . 38

11. IANA Considerations. . . . . . . . . . . . . . . . . . . . 39

11.1. IANA Considerations for MPLS-FTN-STD-MIB . • • • . . . 39

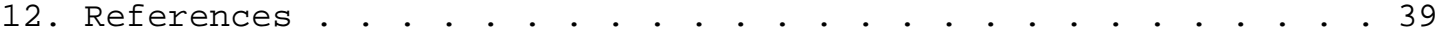

12.1. Normative References . . . . . . . . . . . . . . . . 39

12.2. Informative References . . . . . . . . . . . . . . 40

13. Acknowledgements . . . . . . . . . . . . . . . . . . 41

14. Authors' Addresses . . . . . . . . . . . . . . . . . . . . . 41

15. Full Copyright Statement . . . . . . . . . . . . . . . 42

\section{Introduction}

This memo defines a portion of the Management Information Base (MIB) for use with network management protocols in the Internet community. In particular, it describes managed objects for specifying Forwarding Equivalence Class (FEC) to Next Hop Label Forwarding Entry (NHLFE) mappings and corresponding actions for Multiprotocol Label Switching (MPLS) .

At the ingress of an MPLS network, packets entering the MPLS domain are assigned to an FEC. Those packets belonging to an FEC are associated with an NHLFE (i.e., MPLS label) via the FEC-to-NHLFE (FTN) mapping [RFC3031]. This relationship defines how ingress LSRS will impose MPLS labels onto incoming packets. It also defines how egress LSRs will decapsulate the MPLS shim header from MPLS packets.

Conceptually, some of the FTN table functionality could be implemented using the Forwarding Information Base (FIB) to map all packets destined for a prefix to an LSP. However, this mapping is coarse in nature.

Similar functionality is already being used in other contexts such as security filters, access filters, and RSVP flow identification. All of these require various combinations of matching based on IP header and upper-layer header information to identify packets for a particular treatment. When packets match a particular rule, a corresponding action is executed on those packets. For example, two popular actions to take when a successful match is identified are allowing the packet to be forwarded or to discard it. However, other 
actions are possible, such as modifying the TOS byte, or redirecting a packet to a particular outgoing interface. In the context of MPLS, the possible actions performed by an NHLFE are to redirect packets to either an MPLS Label Switched Path (LSP) or an MPLS Traffic Engineered (TE) Tunnel.

This document attempts to consolidate the various matching requirements and associated action options needed for MPLS into a single specification.

2. Terminology

Although all of the terminology used in this document is either covered in the MPLS Architecture [RFC3031] or in the SNMP Architecture [RFC3411], it is informational to define some immediately pertinent acronyms/terminology here.

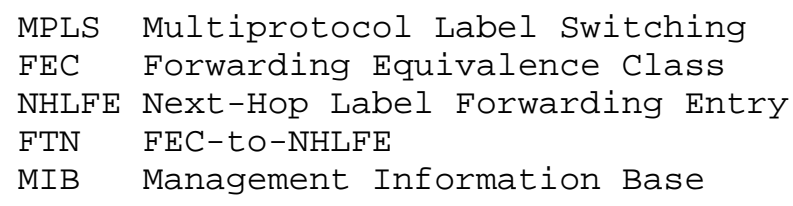

3. Conventions Used In This Document

The key words "MUST", "MUST NOT", "REQUIRED", "SHALL", "SHALL NOT", "SHOULD", "SHOULD NOT", "RECOMMENDED", "MAY", and "OPTIONAL" in this document are to be interpreted as described in BCP 14, RFC 2119 [RFC2119] .

4. The Internet-Standard Management Framework

For a detailed overview of the documents that describe the current Internet-Standard Management Framework, please refer to section 7 of RFC 3410 [RFC3410].

Managed objects are accessed via a virtual information store, termed the Management Information Base or MIB. MIB objects are generally accessed through the Simple Network Management Protocol (SNMP). Objects in the MIB are defined using the mechanisms defined in the Structure of Management Information (SMI). This memo specifies a MIB module that is compliant to the SMIV2, which is described in STD 58, RFC 2578 [RFC2578], STD 58, RFC 2579 [RFC2579] and STD 58, RFC 2580 [RFC2580]. 


\section{Outline}

This MIB module resides on any LSR which does the FEC-to-NHLFE mapping in order to map traffic into the MPLS domain. This MIB module consists of three tables:

- mplsfTNTable defines the rule base against which incoming packets are matched and defines the actions to be taken on matching packets;

- mplsftNMaptable defines the application of these rules to specific interfaces;

- mplsfTNPerfTable provides performance counters for every entry in mplsfTNTable that is active on one or more interfaces, on a perinterface basis.

\section{1. mplsftnTable}

This table allows FEC to NHLFE mappings to be specified. Each entry in this table (also referred to as an "FTN entry" in this document) defines a rule to be applied to incoming packets (on interfaces that the entry is activated on using mplsfTNMapTable as explained in Section 5.2) and an action to be taken on matching packets. mplsfTNTable allows 6-tuple matching rules based on one or more of source address range, destination address range, source port range, destination port range, IPv4 Protocol field [RFC791] or IPv6 nextheader field [RFC2460], and the DiffServ Code Point (DSCP, [RFC2474]) to be specified. Packet redirection is based on an action pointer which points either at an mplsXCEntry in MPLS-LSR-STD-MIB [RFC3813] when the NHLFE is a non-TE LSP, or at an mplsTunnelentry in MPLS-TESTD-MIB [RFC3812] when the NHLFE is the origin of a TE tunnel.

\subsubsection{Advantages of Address Ranges Over CIDR Prefixes}

One possible way of specifying a set of addresses as part of an FTN rule is to use CIDR prefixes [RFC1519]. We have instead chosen to allow FTN rules to be expressed in terms of address ranges in mplsfTNTable because they have the following advantages.

- The number of CIDR prefixes needed to represent some address ranges is very large. For example, we need the following 6 CIDR prefixes to represent the range of addresses [192.0.2.0192.0.2.62]: 192.0.2.0/27, 192.0.2.32/28, 192.0.2.48/29, 192.0.2.56/30, 192.0.2.60/31, and 192.0.2.62/32. A rule such as "redirect all packets with a source address in the range [192.0.2.0-192.0.2.62] and destination address in the range [192.0.2.128-192.0.2.190] to tunnel \#2" would require the creation 
of 36 conceptual rows in mplsfTNTable if the rules were expressed as CIDR prefixes, but only a single conceptual row would be required if we used address ranges instead.

- Every CIDR prefix can be expressed as a single equivalent address range.

- A particular implementation is free to translate the address ranges specified in mplsFTNTable internally to equivalent CIDR prefixes, if it so chooses. However, given that powerful range matching algorithms are available, many implementations may prefer to implement these directly.

5.2. mplsfTNMapTable

This table provides the capability to activate or map FTN entries defined in mplsfTNTable to specific interfaces in the system. Packets received on an interface are compared against FTN entries in the order in which entries are applied to the interface.

\subsubsection{Indexing Requirements}

The indexing structure of mplsfTNMapTable was designed to satisfy the following requirements.

- We must be able to insert a new entry into an existing list of entries on an interface with a single SET operation. Thus, we must be able to support an insertion operation that does not require manual reindexing of existing entries.

- A management application must be able to traverse entries that have been applied to a particular interface in the order of application. The number of (non-bulk) retrieval operations to obtain this information as dictated by the particular indexing scheme that we choose for mplsfTNMapTable must be no more than that dictated by any other indexing scheme. For example, the indexing scheme must not force the Network Management Application to retrieve all the entries in the table and sift through them offline to obtain this information.

\subsubsection{How the Current Indexing Works}

The natural data-structure for implementing constant time insertions between two existing entries and for supporting in-order traversals is a linked-list.

The chosen indexing structure of mplsfTNMapTable makes the entries in the table behave like items in a linked-list. Each conceptual row 
has an object, mplsfTNMapPrevindex, which is a pointer to the previous entry that is applied to a particular interface. This object is self-adjusting, i.e., its value is automatically adjusted by the agent, if necessary, after an insertion or deletion operation.

This indexing scheme provides a mechanism to 'insert' an FTN entry between two existing entries already applied on an interface. This is done by specifying the entry after which a new entry should be inserted in mplsFTNMapPrevIndex.

Using this linked-list structure, one can retrieve FTN entries in the order of application on a per-interface basis as follows:

- To determine the first FTN entry on an interface with index ifIndex, perform a GETNEXT retrieval operation on mplsFTNMapRowStatus.ifIndex.0.0; the returned object, if one exists, is (say) mplsfTNMapRowStatus.ifIndex.0.n (mplsfTNMapRowStatus is the first accessible columnar object in the conceptual row). Then, the index of the first FTN entry applied on this interface is $n$.

- To determine the FTN entry applied to an interface after the one indexed by $n$, perform a GETNEXT retrieval operation on mplsfTNMapRowStatus.ifIndex.n.0. If such an entry exists, the returned object would be of the form mplsfTNMapRowStatus.ifIndex.n.m. Then, the index of the next FTN entry applied on this interface is $\mathrm{m}$.

- If the FTN entry indexed by $\mathrm{n}$ is the last entry applied to the interface with index ifIndex, then the object returned would either be:

1. mplsfTNMapRowStatus.ifIndexNext.0.k, where ifIndexNext is the index of the next interface in ifTable to which an FTN entry has been applied, in which case $\mathrm{k}$ is the index of the first FTN entry applied to the interface with index ifIndexNext;

or:

2. mplsfTNMapStorageType.firstIfIndex.0.p, if there are no more entries in mplsfTNMapTable, where firstifindex is the first entry in ifTable to which an FTN entry has been mapped.

The above steps can be used to retrieve all the applied entries on a per-interface basis in application order. Note that the number of retrieval operations is equal to the number of applied FTN entries (i.e., the minimum number of GETNEXT operations needed using any indexing scheme). 
Also note that we could not have created this linked-list structure using a 'next' pointer object instead of the 'previous' pointer object that we chose because this would not allow us to determine the first FTN entry that has been mapped to a specific interface using a single SNMP (non-bulk) retrieval operation.

The use of this indexing structure is further illustrated using an example in section 7 .

\section{3. mplsfTNPerfable}

If an FTN entry has been applied to one or more interfaces, this table provides high-capacity performance counters to monitor each such FTN entry on a per-interface basis.

6. Avoiding Retrieval-Modification Interactions

The problem of an ongoing traversal or retrieval operation on an SNMP table being affected by a concurrent modification operation on that table is not unique to this MIB module. However, it is useful to note that a cautious application can keep track of the state of the modifiable tables in this MIB module using the objects mplsFTNTableLastChanged and mplsFTNMapTableLastChanged.

For instance, before performing a traversal of mplsfTNMapTable, the application should retrieve the value of mplsFTNMapTableLastchanged. Each subsequent GETNEXT operation on the table should include this object as well. For example, GETNEXT(mplsFTNMapTableLastChanged.0, mplsFTNMapRowStatus.ifIndex.n.0) can be used to:

- Determine the FTN entry after the one indexed by $\mathrm{n}$ (in linked-list order) mapped to the interface with index ifIndex, as explained in Section 5.2.2;

- Verify that the value of mplsfTNMapTable has not been modified during the retrieval process by comparing the value of mplsFTNMapTableLastChanged retrieved by this operation with the value retrieved before the traversal was begun.

Using this technique, an application can ensure the validity of the retrieved information with minimal overhead. This is particularly important while retrieving information from frequently modified tables. 
7. Example Illustrating MIB Module Components

In this section, we use an example to illustrate how the objects defined in MPLS-FTN-STD-MIB work together to perform FEC to NHLFE mapping .

Note that for the various table entries involved in this example, we only show the objects that help illustrate each case.

\subsection{Sample FTN Rules}

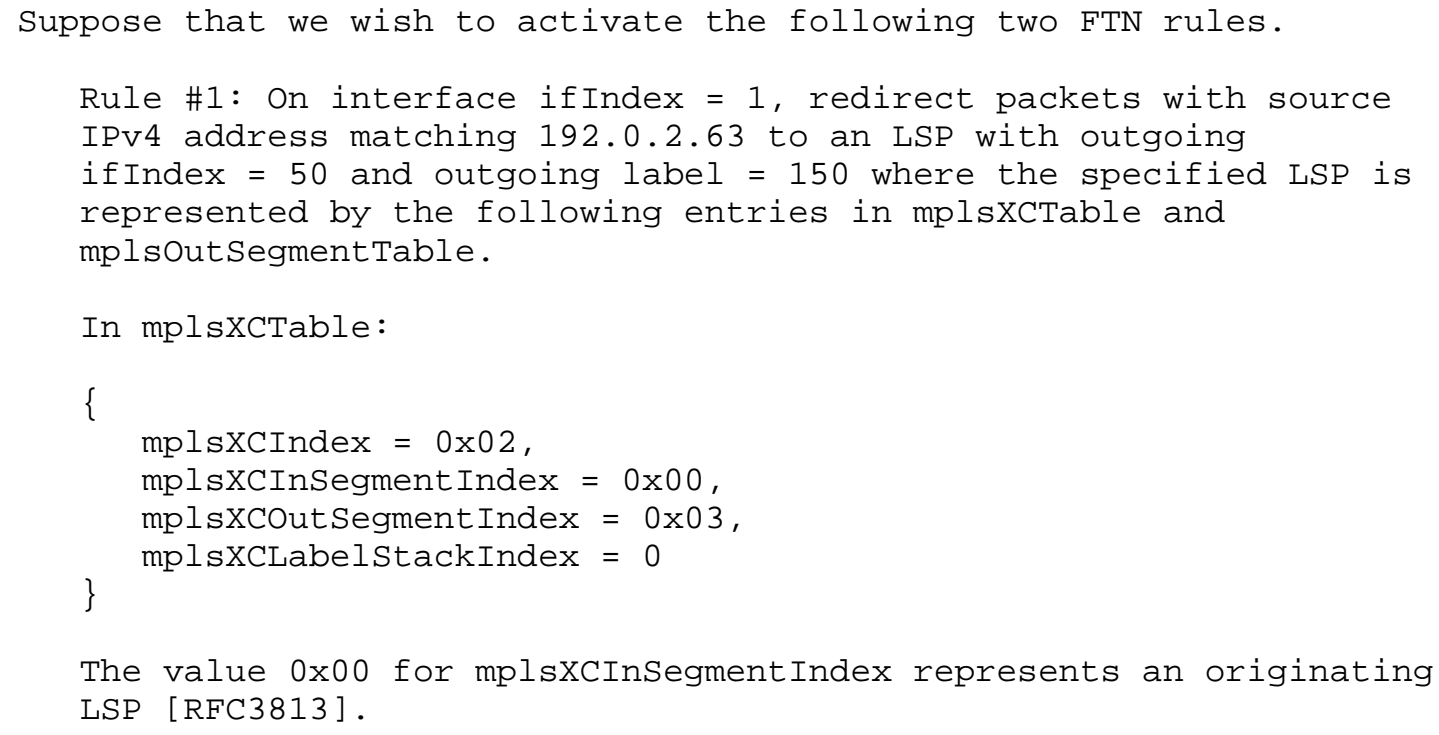


\{

mplstunnelIndex $=4$, -- primary tunnel mplstunnelinstance $=0$, mplsTunnelIngressLSRID $=192.0 .2 .1$, mplstunnelEgressLSRID = 192.0.2.2

\}

7.2. Creating FTN Entries and Applying them to Interfaces

The action "redirect packets with source IPv4 address matching 192.0.2.63 to an LSP with outgoing ifIndex $=50$ and outgoing label= 150" in Rule \#1 can be implemented by the following entry in mplsfTNTable:

\{

mplsFTNIndex $=1$, mplsFTNDescr = "Rule \#1", -- source address only mplsFTNMask $=0 \times 80$, mplsFTNAddrType $=$ ipv4, mplsFTNSourceAddrMin $=192.0 .2 .63$, mplsFTNSourceAddrMax $=192.0 .2 .63$, mplsFTNActionType = redirectLsp (1), mplsFTNActionPointer $=$ mplsXCLspId.1.2.1.0.1.3 \}

This indicates to which LSP the LSR should redirect packets by setting mplsFTNActionPointer to the first accessible columnar object instance in mplsXCEntry that corresponds of the LSP to use, in this case mplsXCLspId.1.2.1.0.1.3.

This action is then activated on "interface ifIndex $=1$ " by the following entry in mplsFTNMapTable to complete the implementation of Rule \#1:

\{ -- apply rule to interface ifIndex $=1$ mplsFTNMapIndex $=1$, -- first FTN entry on this interface mplsFTNPrevIndex $=0$, -- index of current entry in mplsfTNTable, i.e., Rule \#1 mplsFTNMapCurrIndex $=1$

\}

The action "redirect packets with destination IPv4 addresses in the range [192.0.2.32, 192.0.2.96] to tunnel \#4" in Rule \#2 can be implemented by the following entry in mplsfinTable: 
\{

mplsFTNIndex $=2$, mplsfTNDescr $=$ "Rule \#2", -- destination address only mplsFTNMask $=0 \times 40$, mplsFTNAddrType $=$ ipv 4 mplsFTNDestAddrMin = 192.0.2.32, mplsFTNDestAddrMax = 192.0.2.96, mplsFTNActionType = redirectTunnel (2), mplsfTNActionPointer $=$ mplstunnelName.4.0.3221225985.3221225986 \}

where 3221225985 and 3221225986 are representations of the addresses 192.0.2.1 and 192.0.2.2, respectively, as Unsigned32 (the underlying data type) entities.

This rule needs to be activated on "interface ifIndex = 1 " after Rule \#1 which was previously activated on this interface. This is done by the following entry in mplsfinMaptable to complete the implementation of Rule \#2:

\{

-- apply rule to interface ifIndex $=1$

mplsFTNMapIndex $=1$,

-- insert after Rule \#1 (mplsfTNIndex = 1)

mplsFTNPrevIndex $=1$,

-- index of current entry in mplsfTNTable, i.e., Rule \#2

\}

mplsFTNMapCurrIndex $=2$

7.3. Mapping an FTN Entry to Multiple Interfaces

Suppose we now wish to activate the following rule:

Rule \#2b: On interface ifIndex $=2$, redirect packets with destination IPv4 addresses in the range [192.0.2.32, 192.0.2.96] to tunnel \#4.

Notice that the FEC and corresponding action associated with this rule (i.e., "redirect packets with destination IPv4 addresses in the range [192.0.2.32, 192.0.2.96] to tunnel \#4") are the same as that associated with Rule \#2. Hence, we can reuse the existing entry with mplsfTNIndex $=2$ from mplsfTNTable.

However, we have to create the following new entry in mplsFTNMapTable to activate this FTN entry as the first one on the interface with ifIndex $=2$. 
\{

-- apply rule to interface ifIndex $=2$

mplsFTNMapIndex $=2$,

-- first FTN entry on this interface

mplsFTNPrevIndex $=0$,

-- index of current entry in mplsftntable

\}

mplsFTNMapCurrIndex $=2$

7.4. Inserting an Entry Into Existing List

At a later point, suppose that we wish to introduce the following Rule between Rules \#1 and \#2.

Rule \#3: On interface ifIndex $=1$, redirect all packets with destination IPV4 address matching the prefix 192.0.2.32/28 to tunnel \#3, where the tunnel we wish to redirect traffic to is represented by the following entry in mplstunnelTable:

\{

mplstunnelindex $=3$,

-- primary tunnel

mplstunnelinstance $=0$,

mplstunnelingressLSRID $=192.0 .2 .3$,

\}

mplstunnelEgressLSRID $=192.0 .2 .4$

Note that the ordering of the rules on a particular interface is critical since the range of addresses specified in Rule \#3 is a subset of the ones specified in Rule \#2.

Without the linked-list style insertion feature supported by mplsfTNMapTable, we would possibly have had to reindex existing entries (or plan for such changes by leaving sufficient gaps between indexes, something that only postpones the problem). With the existing tables, we solve this problem by creating the following entries.

We implement the phrase "redirect all packets with destination IPv4 address matching the prefix $1.4 .0 .0 / 16$ to tunnel \#3" in Rule \#3 by creating the following entry in mplsfTNTable: 
\{

mplsFTNIndex $=3$, mplsfTNDescr = "Rule \#3", -- destination address only mplsFTNMask $=0 \times 40$, mplsFTNAddrType $=$ ipv4, -- address range equivalent to CIDR prefix 192.0.2.32/28 mplsFTNDestAddrMin $=192.0 .2 .32$, mplsFTNDestAddrMax $=192.0 .2 .47$, mplsFTNActionType = redirectTunnel, \}

mplsFTNActionPointer $=$ mplstunnelName.3.0.3221225987.3221225988

where 3221225987 and 3221225988 are representations of the addresses 192.0.2.3 and 192.0.2.4, respectively, as Unsigned32 (the underlying data type) entities.

We next insert this rule in mplsfTNMapTable just after Rule \#1 as follows :

\{ -- apply rule to interface ifIndex $=1$ mplsFTNMapIndex $=1$, -- insert after Rule \#1 (mplsFTNIndex = 1) mplsFTNPrevIndex $=1$, -- index of current entry in mplsfTNTable i.e., Rule \#3 mplsFTNMapCurrIndex $=3$ \}

After the insertion of Rule \#3 in mplsfTNMapTable, the 'previous' pointer object mplsFTNMapPrevIndex of the next entry (corresponding to Rule \#2) adjusts automatically to point to this entry.

Note that, of the existing entries in the table, the only one that is impacted by an insertion operation is the entry on that particular interface immediately after the newly inserted one, if one exists. None of the other entries in mplsfTNMapTable are impacted. For instance, in this particular example, when the entry for Rule \#3 was inserted between those for Rules \#1 and \#2, the entries for Rules \#1 and \#2b were not impacted. 


\subsection{Pictorial Tabular Relationship}

At this point, the relationship between different table entries can be represented pictorially as follows. For each conceptual row instance, we show the table that it belongs to, along with its indices in parentheses. (Note that various conceptual rows are depicted in a way that is convenient for showing the interrelationships and are not necessarily in lexicographical order.)

iftable, The Interfaces Group MIB [RFC2863]:

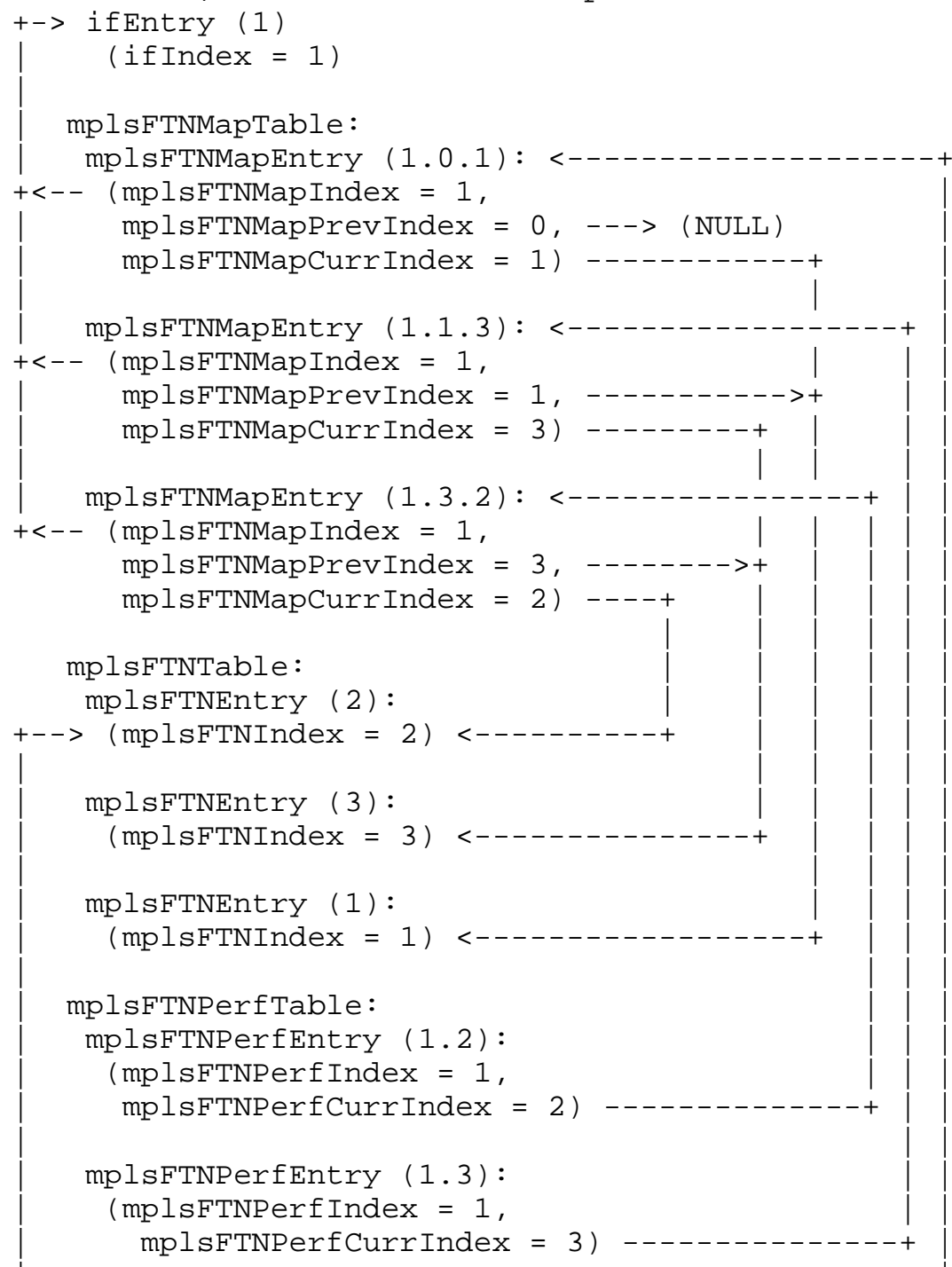




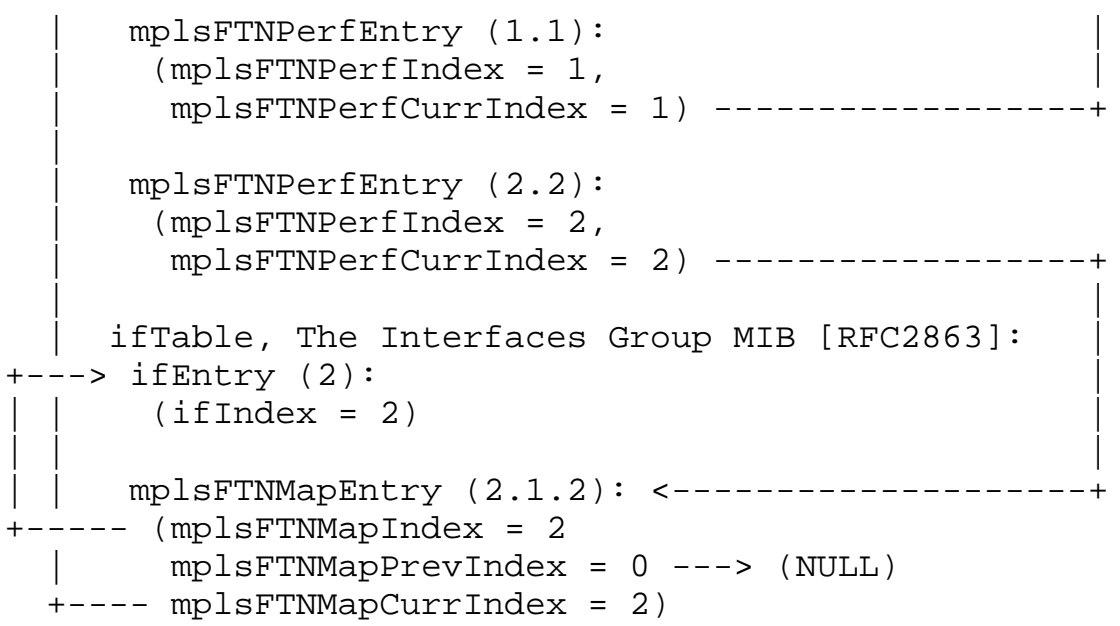

\subsection{Deleting an Entry}

Let us next look at how we can remove the recently applied Rule \#3 and how the existing conceptual rows behave in this situation.

The conceptual row corresponding to the application of Rule \#3 to interface ifIndex $=1$ has the following index values: mplsFTNMapIndex $=1$, mplsfTNMapPrevindex $=1$, and mplsfTNMapCurrIndex $=3$. To delete this conceptual row, the Network Management Application performs a SET operation setting the object instance mplsFTNMapRowstatus.1.1.3 to the value destroy(6). The agent then destroys this conceptual row. It also automatically adjusts the object instance of mplsfTNMapPrevIndex corresponding to Rule \#2 from the value 3 (i.e., pointing to the recently destroyed Rule \#3) to the value 1 (i.e., to Rule \#1).

At this point, the rules applied to interface ifIndex $=1$ are Rule \#1 and Rule \#2, in that order. The relationship between different table entries can be represented pictorially as follows. 


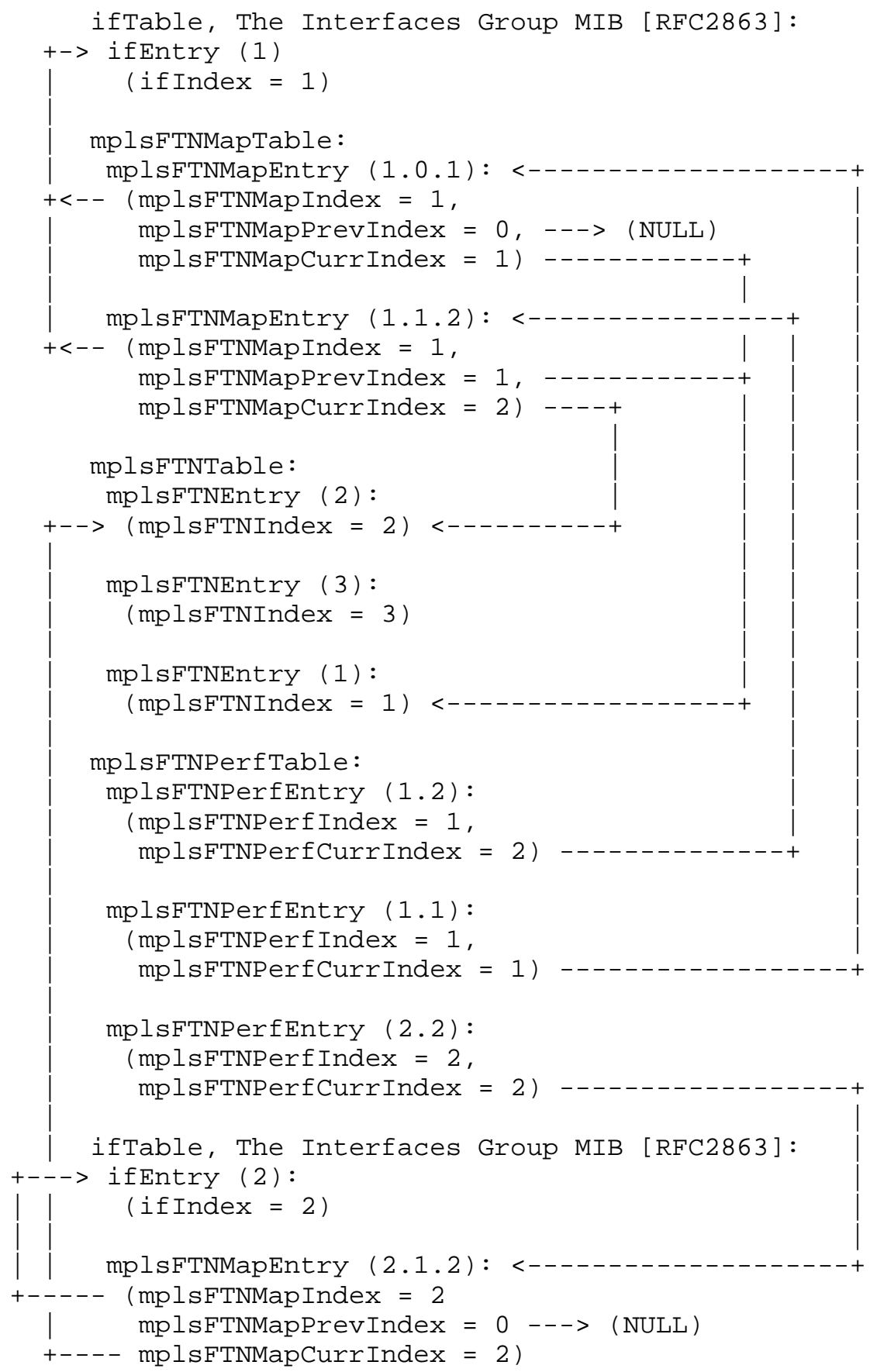


Note that the FTN entry for Rule \#3 still exists in mplsfTNTable at this point but is not referenced by any conceptual row in mplsFTNMapTable or mplsFTNPerftable.

Also note that the deletion of an entry from mplsfTNMapTable only impacts the entry on that particular interface immediately after the deleted entry, if one exists. None of the other conceptual rows in mplsfTNMapTable are impacted. For instance, in this particular example, when the entry for Rule \#3 was deleted, the entries for Rules \#1 and \#2b were not impacted.

8. The Use of RowPointer

RowPointer is a textual convention used to identify a conceptual row in a conceptual table in a MIB by pointing to the first accessible object. In this MIB module, in mplsfTNTable, the RowPointer object mplsFTNActionPointer indicates the LSP or TE Tunnel to redirect packets matching an FTN entry to. This object MUST point to the first instance of the first accessible columnar object in the appropriate conceptual row in order to allow the manager to find the appropriate corresponding entry in either MPLS-LSR-STD-MIB [RFC3813] or MPLS-TE-STD-MIB [RFC3812]. If this object returns zeroDotzerok, it implies that there is no currently defined action that is associated with that particular FTN entry.

9. MPLS-FTN-STD-MIB Definitions

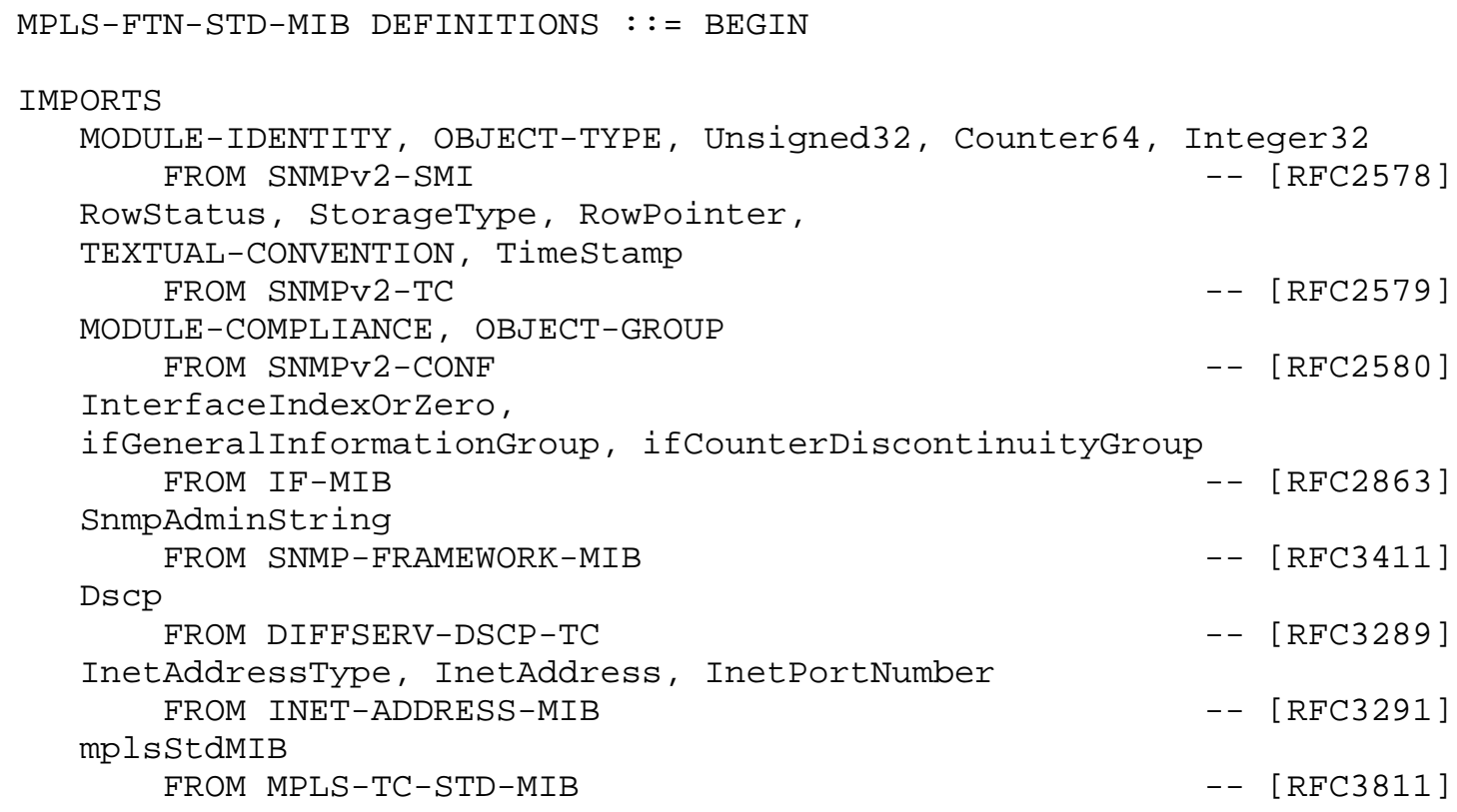


;

mPlsFTNSTdMIB MODULE-IDENTITY

LAST-UPDATED "200406030000z" -- June 6, 2004

ORGANIZATION "Multiprotocol Label Switching (MPLS) Working Group" CONTACT-INFO

"

Thomas D. Nadeau

Postal: Cisco Systems, Inc. 250 Apollo Drive

Chelmsford, MA 01824

Tel: +1-978-244-3051

Email: tnadeaulcisco.com

Cheenu Srinivasan

Postal: Bloomberg L.P. 499 Park Avenue

New York, NY 10022

Tel: $\quad+1-212-893-3682$

Email: cheenu@bloomberg.net

Arun Viswanathan

Postal: Force10 Networks, Inc. 1440 McCarthy Blvd

Milpitas, CA 95035

Tel: +1-408-571-3516

Email: arunv@forcelonetworks.com

IETF MPLS Working Group email: mpls@uu.net"

DESCRIPTION

"Copyright (C) The Internet Society (2004). The initial version of this MIB module was published in RFC 3814. For full legal notices see the RFC itself or see:

http://www.ietf.org/copyrights/ianamib.html

This MIB module contains managed object definitions for specifying FEC to NHLFE (FTN) mappings and corresponding performance for MPLS."

-- Revision history.

REVISION

"200406030000Z" -- June 3, 2004

DESCRIPTION

"Initial version issued as part of RFC 3814." 


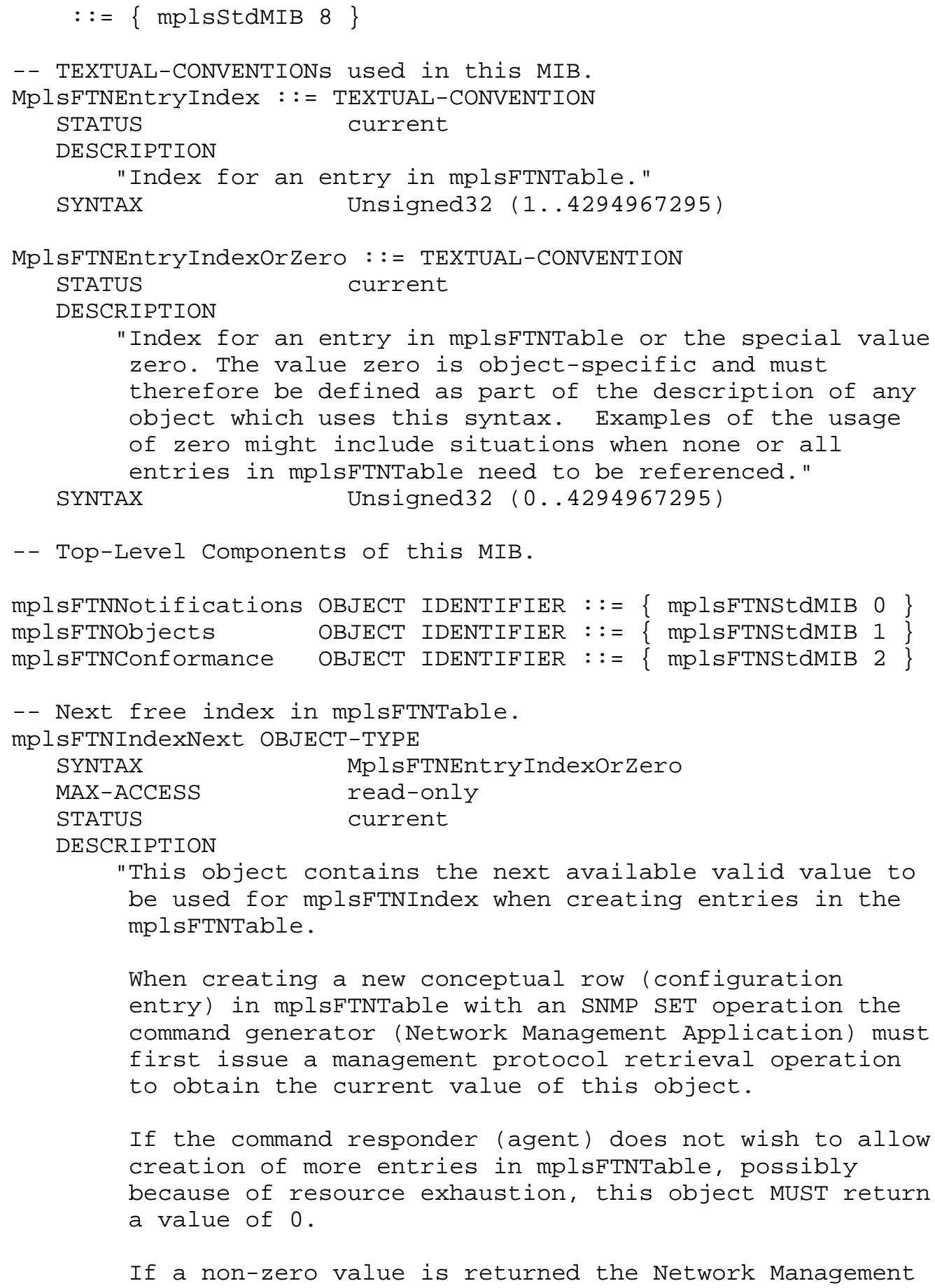


Application must determine whether the value is indeed still unused since two Network Management Applications may attempt to create a row simultaneously and use the same value.

If it is currently unused and the SET succeeds, the agent MUST change the value of this object to a currently unused non-zero value (according to an implementation specific algorithm) or zero (if no further row creation will be permitted).

If the value is in use, however, the SET fails and the Network Management Application must then reread this object to obtain a new usable value." $::=\{$ mplsfTNObjects 1$\}$

-- Last time an object in mplsFTNTable changed. mplsFTNTableLastChanged OBJECT-TYPE SYNTAX Timestamp MAX-ACCESS read-only STATUS current DESCRIPTION

"Indicates the last time an entry was added, deleted or modified in mplsfTNTable. Management stations should consult this object to determine if mplsFTNTable requires their attention. This object is particularly useful for applications performing a retrieval on mplsfTNTable to ensure that the table is not modified during the retrieval operation."

$::=\{$ mplsfinobjects 2$\}$

-- Table of FTN entries. mplsFTNTable OBJECT-TYPE SYNTAX SEQUENCE OF MplsFTNEntry MAX-ACCESS not-accessible STATUS current DESCRIPTION

"This table contains the currently defined FTN entries. This table allows FEC to NHLFE mappings to be specified. Each entry in this table defines a rule to be applied to incoming packets (on interfaces that the FTN entry is activated on using mplsfTNMapTable) and an action to be taken on matching packets

(mplsfTNActionPointer).

This table supports 6-tuple matching rules based on one or more of source address range, destination address range, source port range, destination port range, IPv4 
Protocol field or IPv6 next-header field and the

DiffServ Code Point (DSCP) to be specified.

The action pointer points either to instance of mplsXCEntry in MPLS-LSR-STD-MIB when the NHLFE is a non$\mathrm{TE}$ LSP, or to an instance of mplsTunnelEntry in the MPLS-TE-STD-MIB when the NHLFE is an originating TE tunnel."

REFERENCE

"J. Postel, Internet Protocol, RFC 791, STD 5, September 1981

Deering, S., and R. Hinden, Internet Protocol, Version

6 (IPv6) Specification, RFC 2460, December 1998

Nichols, K, Blake, S., Baker, F. and D. Black, Definition of the Differentiated Services Field (DS Field) in the IPv4 and IPv6 Headers, RFC 2474, December 1998

Srinivasan, C., A. Viswanathan, and T. Nadeau, MPLS Label Switch Router Management Information Base, RFC 3813

Srinivasan, C., A. Viswanathan, and T. Nadeau, MPLS Traffic Engineering Management Information Base, RFC 3812"

$::=\{$ mplsfTNObjects 3$\}$

mplsfTNEntry OBJECT-TYPE

SYNTAX MplsFTNEntry

MAX-ACCESS not-accessible

STATUS current

DESCRIPTION

"Each entry represents one FTN entry which defines a rule to compare incoming packets with and an action to be taken on matching packets."

INDEX \{ mplsFTNIndex $::=\quad\{$ mplsftnTable 1$\}$

MplsfTNEntry $::=$ SEQUENCE \{ mplsFTNIndex mplsFTNRowstatus

mplsFTNDescr mplsFTNMask mplsFTNAddrType mplsFTNSourceAddrMin mplsFTNSourceAddrMax

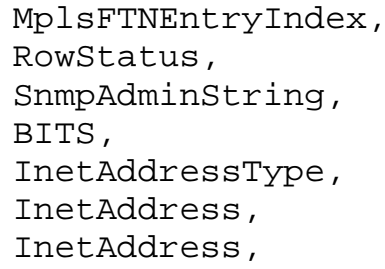




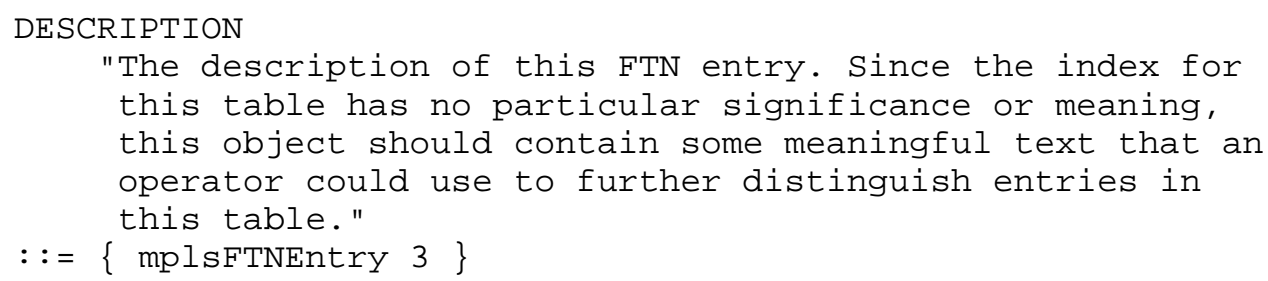




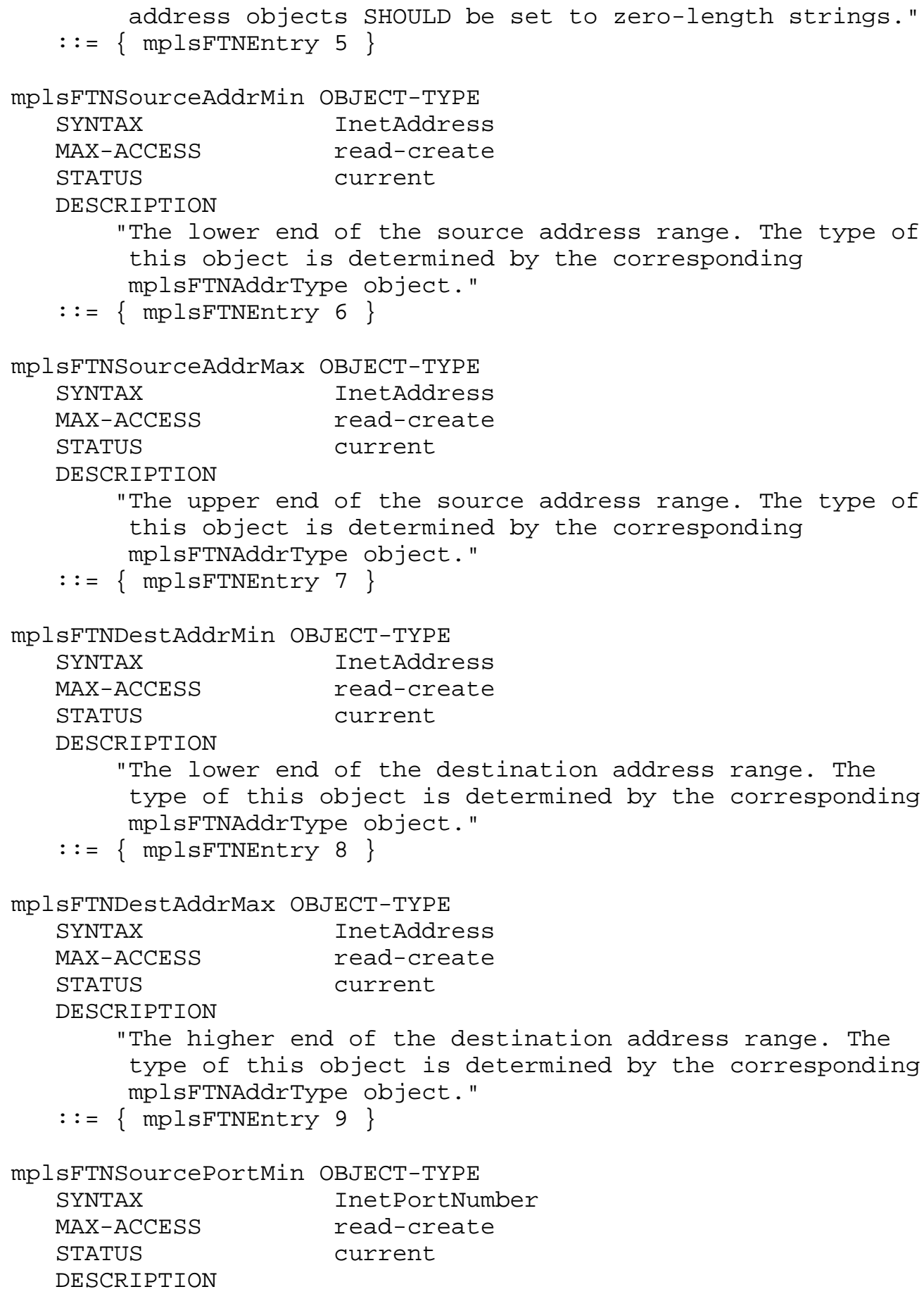




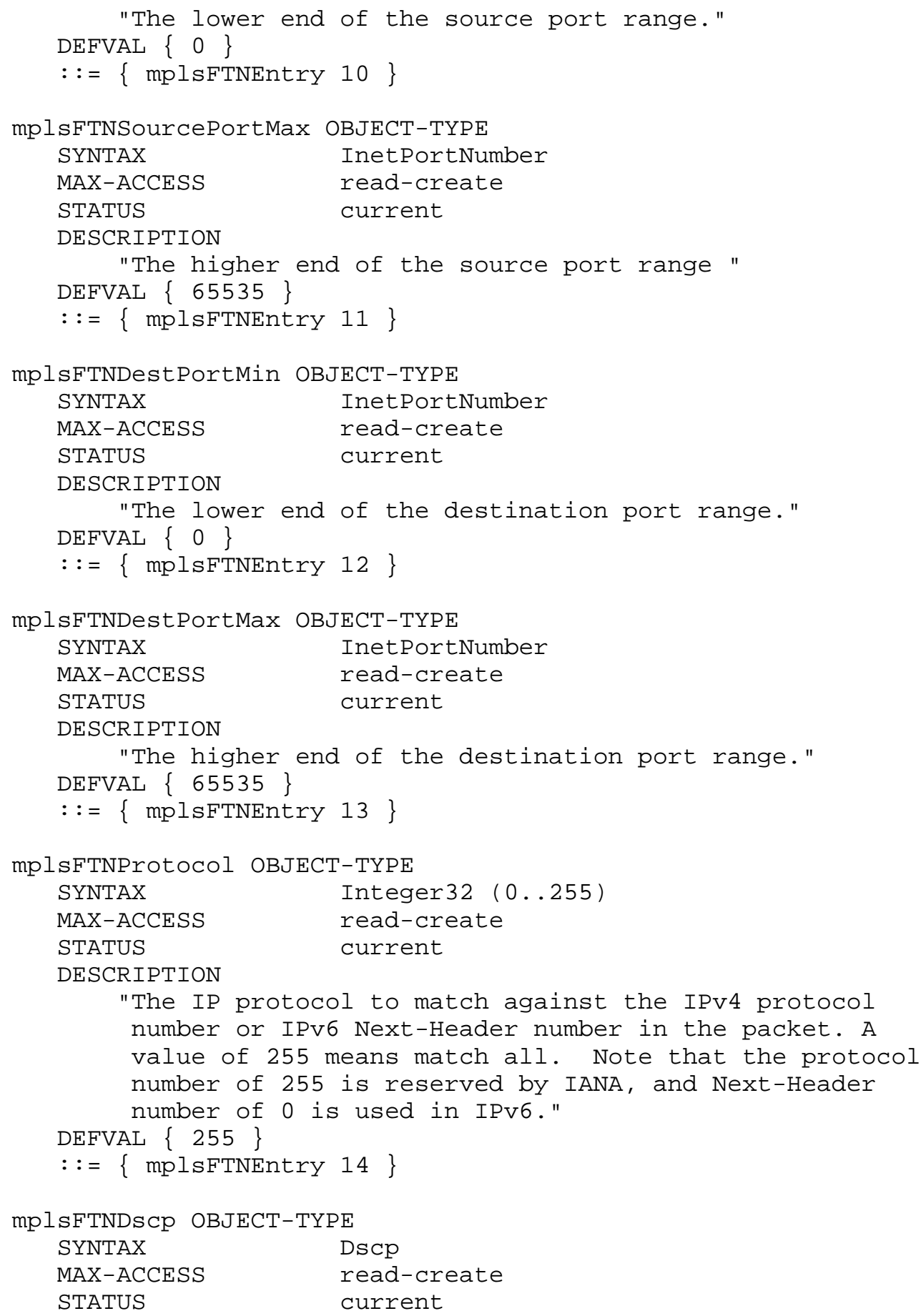




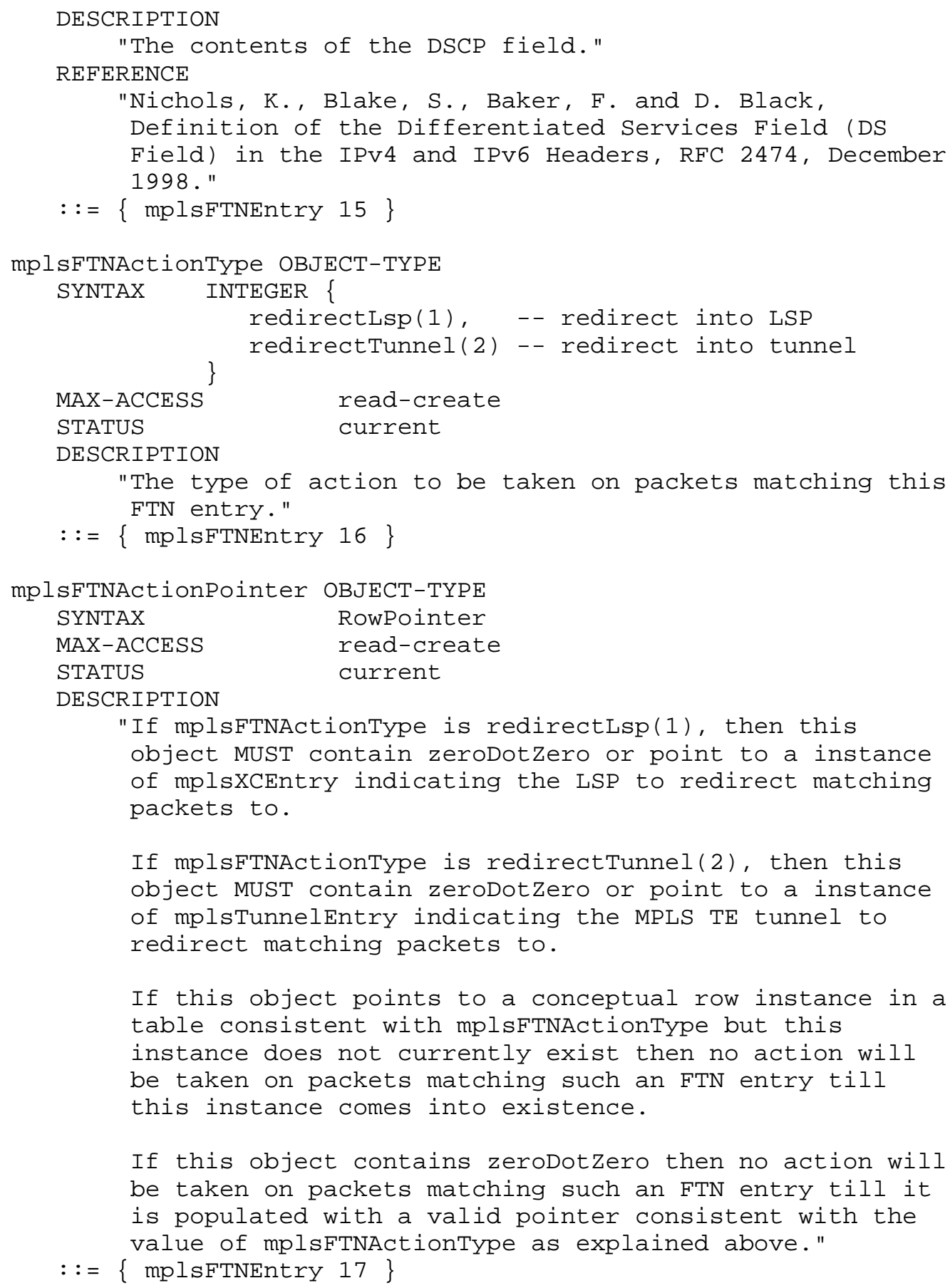




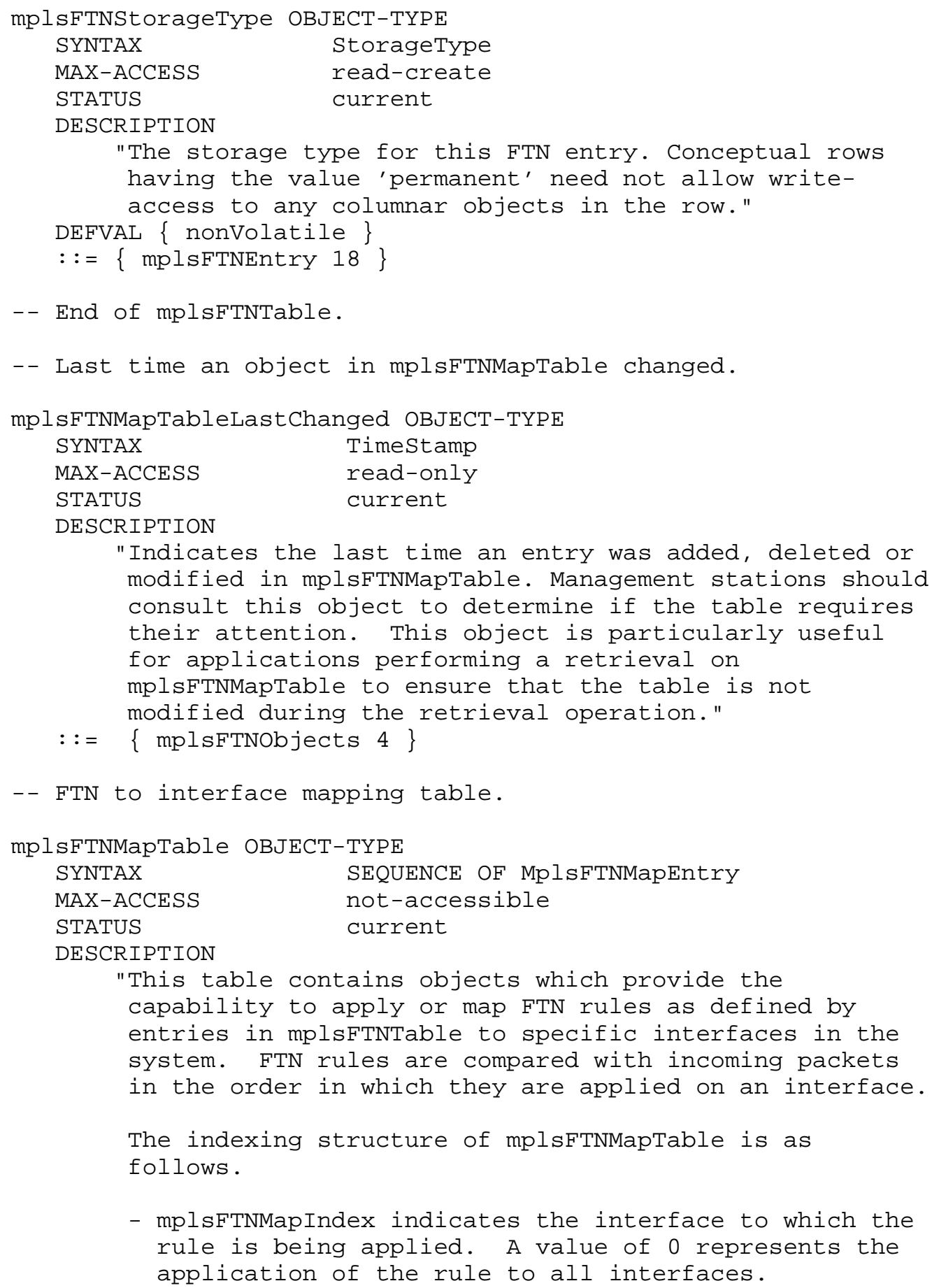


- mplsfTNMapPrevIndex specifies the rule on the interface prior to the one being applied. A value of 0 specifies that the rule is being inserted at the head of the list of rules currently applied to the interface.

- mplsfTNMapCurrIndex is the index in mplsfTNTable corresponding to the rule being applied.

This indexing structure makes the entries in the table behave like items in a linked-list. The object mplsfTNMapPrevindex in each conceptual row is a pointer to the previous entry that is applied to a particular interface. This allows a new entry to be 'inserted' at an arbitrary position in a list of entries currently applied to an interface. This object is selfadjusting, i.e., its value is automatically adjusted by the agent, if necessary, after an insertion or deletion operation.

Using this linked-list structure, one can retrieve FTN entries in the order of application on a per-interface basis as follows:

- To determine the first FTN entry on an interface with index ifIndex perform a GETNEXT retrieval operation on mplsFTNMapRowStatus.ifIndex.0.0; the returned object, if one exists, is (say) mplsFTNMapRowStatus.ifIndex.0.n (mplsFTNMapRowstatus is the first accessible columnar object in the conceptual row). Then the index of the first FTN entry applied on this interface is $n$.

- To determine the FTN entry applied to an interface after the one indexed by $n$ perform a GETNEXT retrieval operation on mplsFTNMapRowStatus.ifIndex.n.0. If such an entry exists the returned object would be of the form mplsfTNMapRowStatus.ifIndex.n.m. Then the index of the next FTN entry applied on this interface is $\mathrm{m}$.

- If the FTN entry indexed by $\mathrm{n}$ is the last entry applied to the interface with index ifIndex then the object returned would either be:

1.mplsFTNMapRowStatus.ifIndexNext.0.k, where ifIndexNext is the index of the next interface in 
iftable to which an FTN entry has been applied, in which case $k$ is the index of the first FTN entry applied to the interface with index ifIndexNext;

or :

2.mplsFTNMapStorageType.firstIfIndex.0.p, if there are no more entries in mplsfTNMapTable, where firstIfIndex is the first entry in iftable to which an FTN entry has been mapped.

Use the above steps to retrieve all the applied FTN entries on a per-interface basis in application order. Note that the number of retrieval operations is the same as the number of applied FTN entries (i.e., the minimum number of GETNEXT operations needed using any indexing scheme).

Agents MUST NOT allow the same FTN entry as specified by mplsFTNMapcurrIndex to be applied multiple times to the same interface.

Agents MUST NOT allow the creation of rows in this table until the corresponding rows are created in the mplsfTNTable.

If a row in mplsfTNTable is destroyed, the agent MUST destroy the corresponding entries (i.e., ones with a matching value of mplsfTNCurrIndex) in this table as well."

$::=\{$ mplsFTnObjects 5$\}$

mplsFTNMapEntry OBJECT-TYPE

SYNTAX MplsfTNMapEntry

MAX-ACCESS not-accessible

STATUS

current

DESCRIPTION

"Each conceptual row represents the application of an FTN rule at a specific position in the list of FTN INDEX \{ rules applied on an interface. "

mplsFTNMapIndex, mplsFTNMapPrevIndex, mplsFTNMapCurrIndex

\} $::=\quad\{$ mplsfTnMaptable 1$\}$

MplsfTNMapEntry $::=$ SEQUENCE \{ 


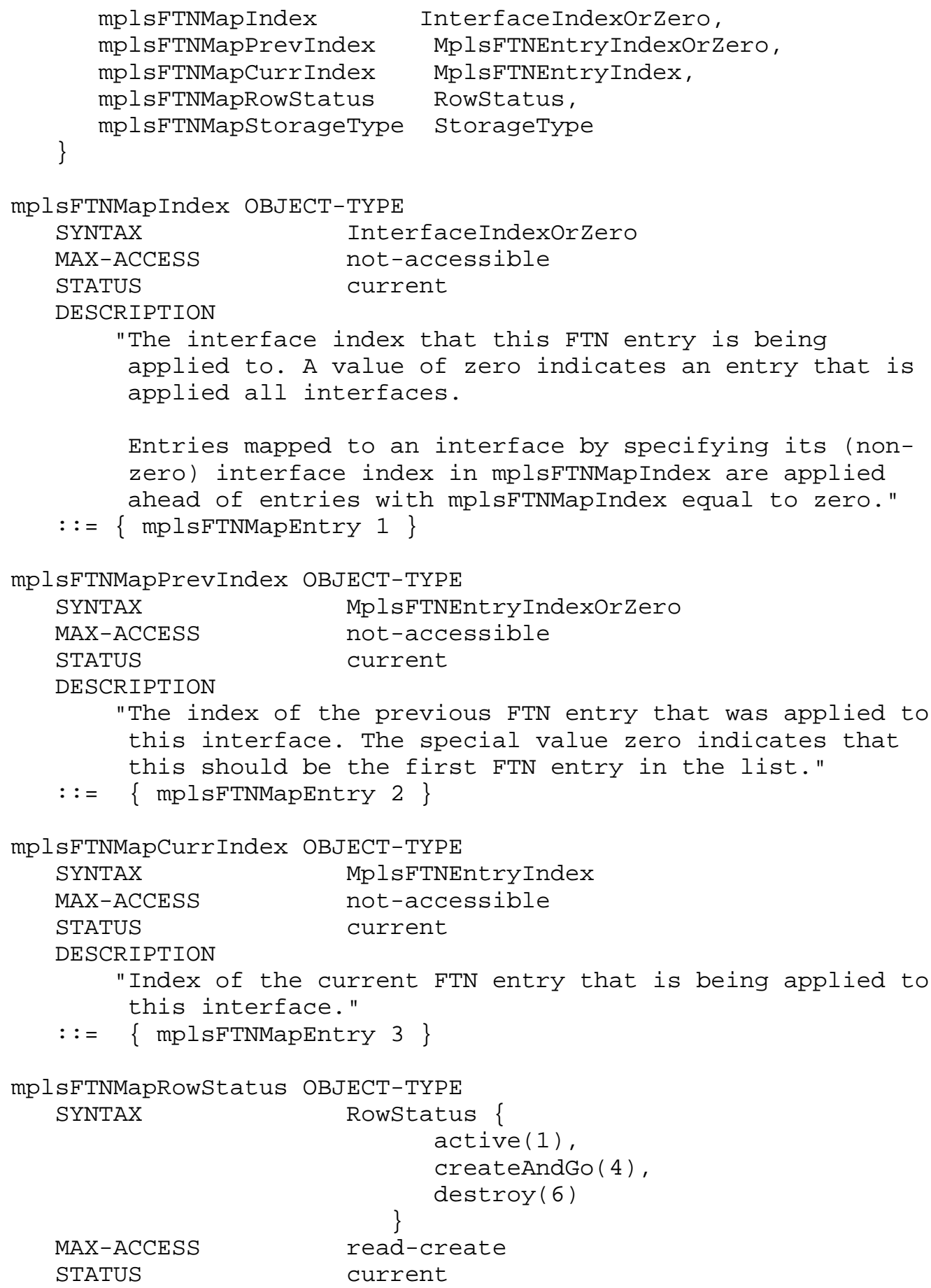




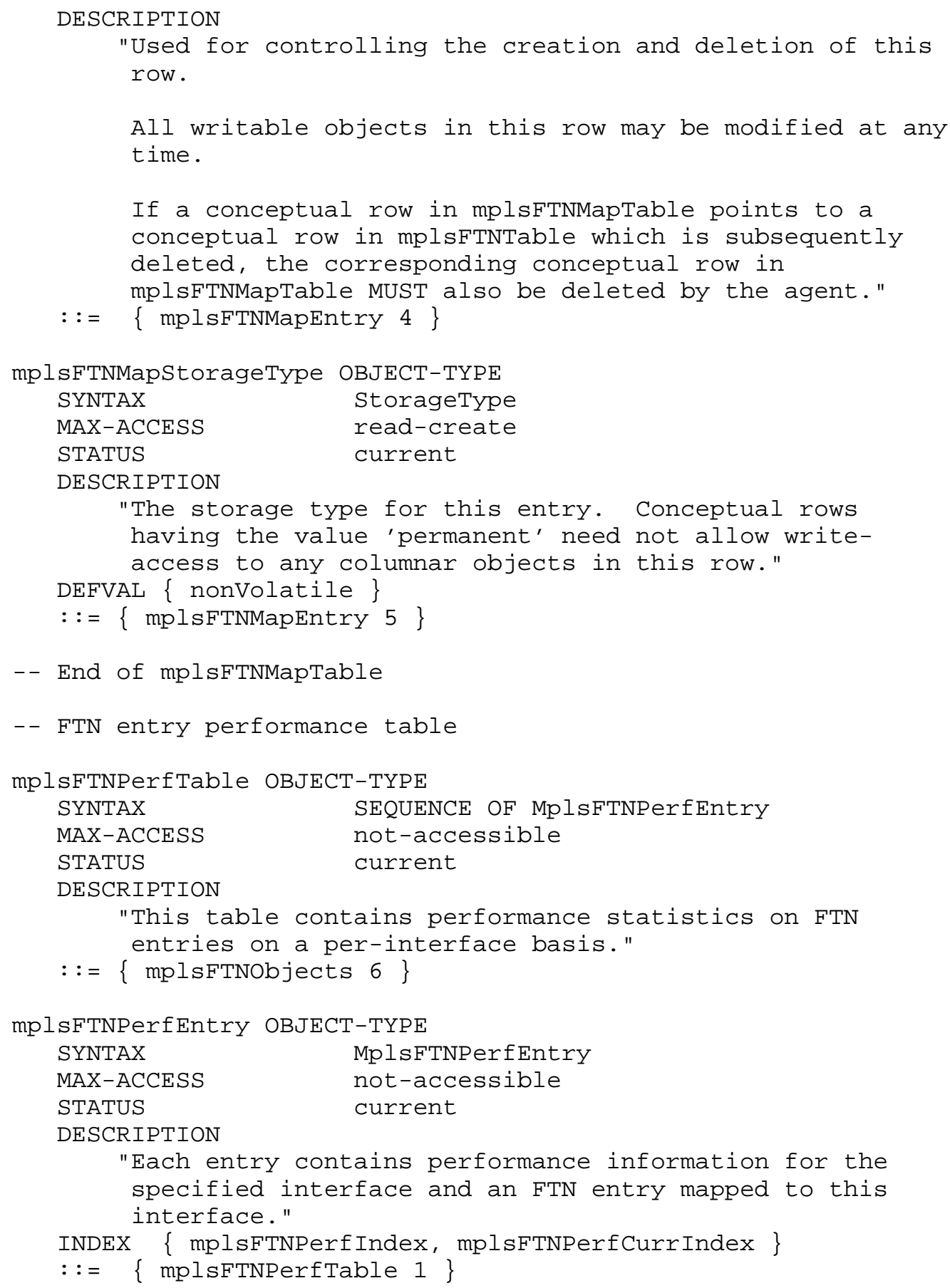




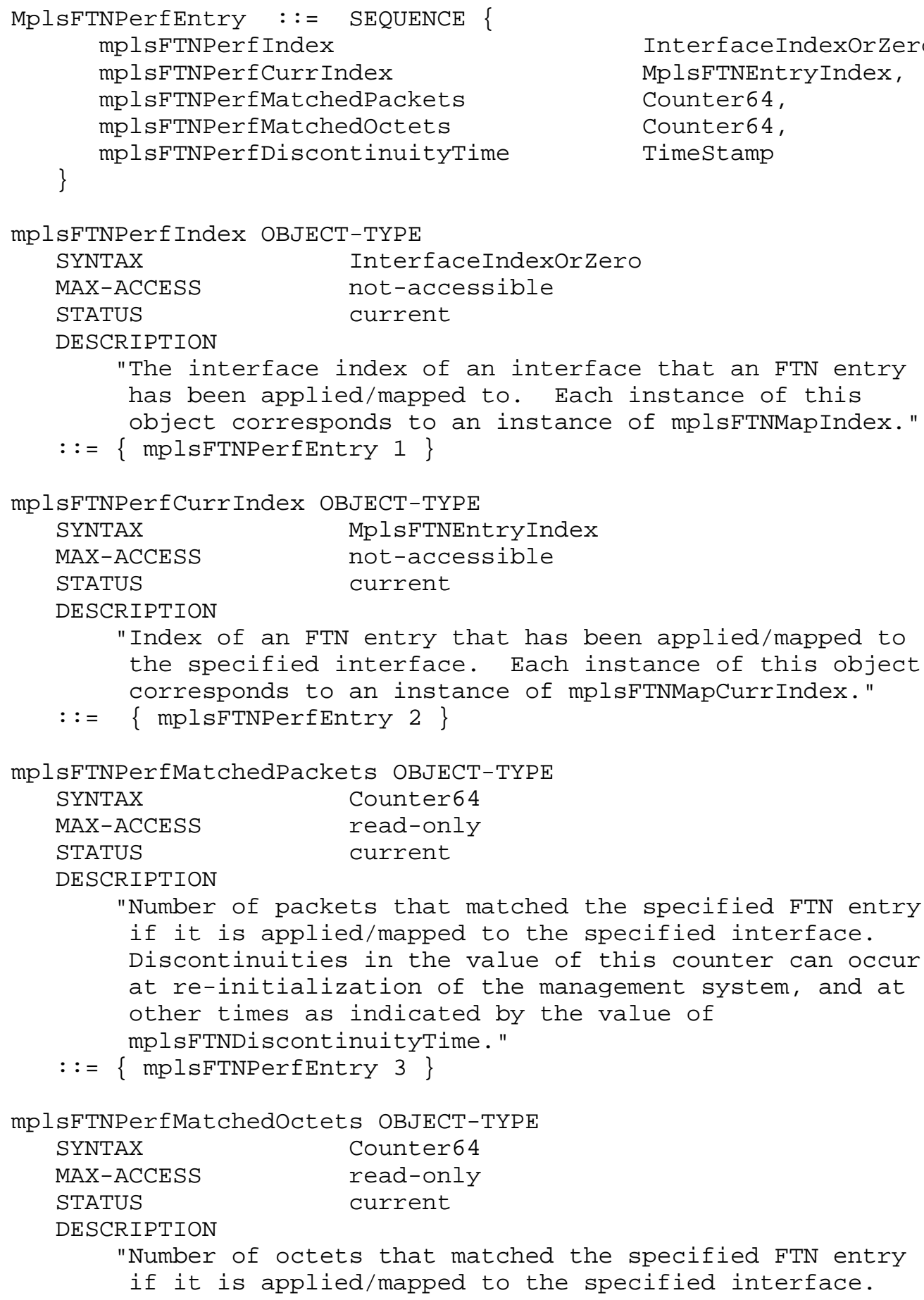




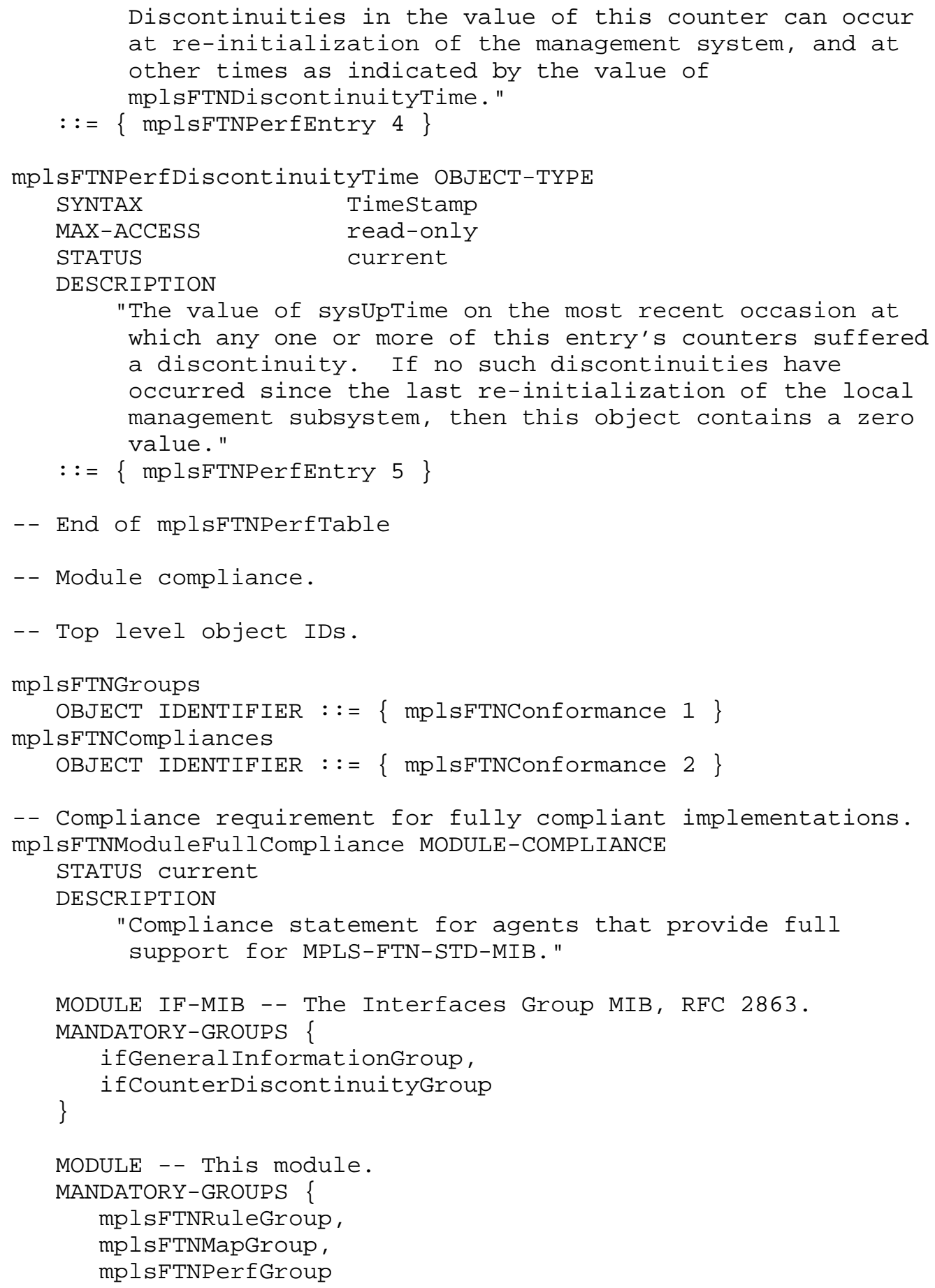




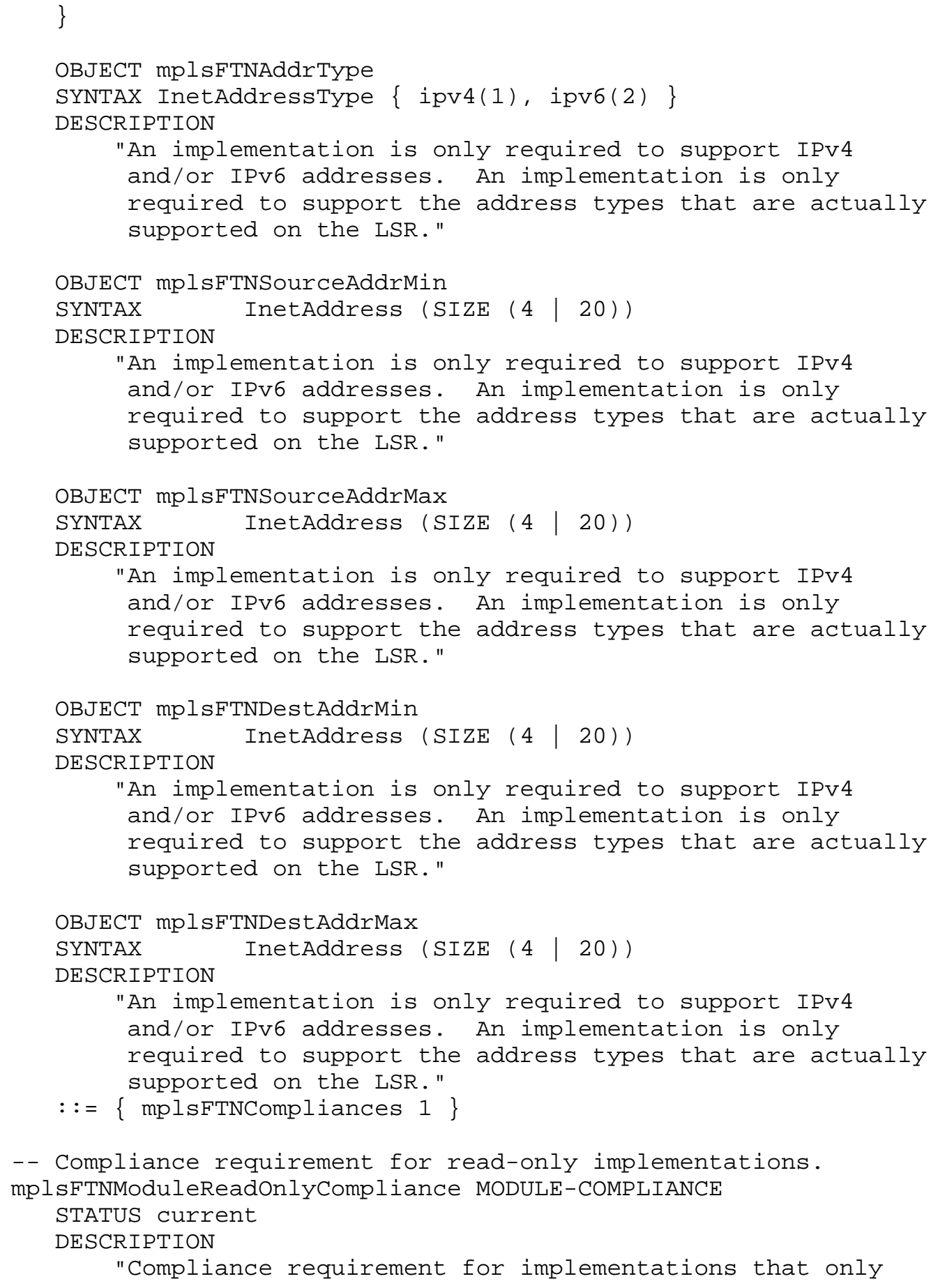




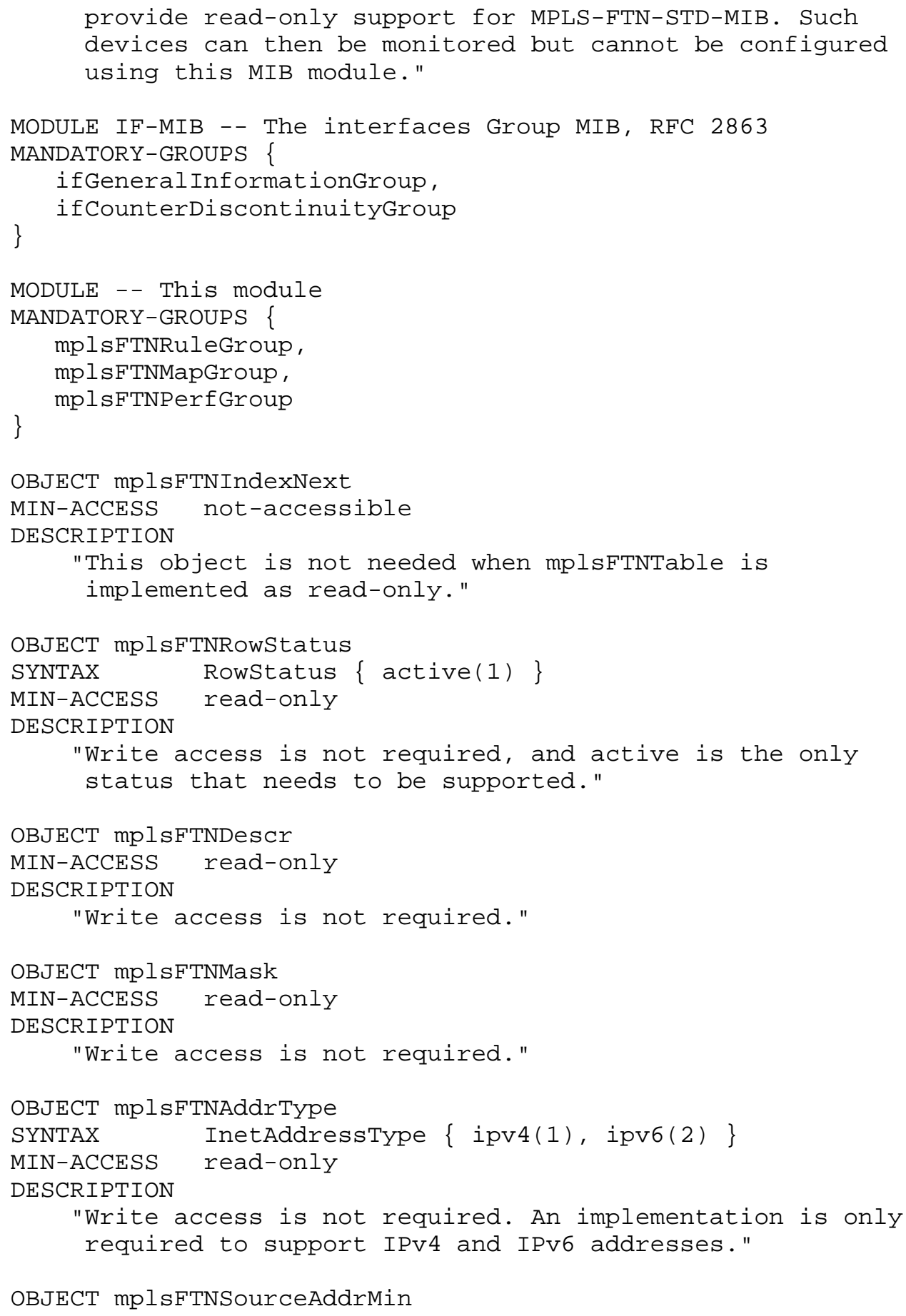




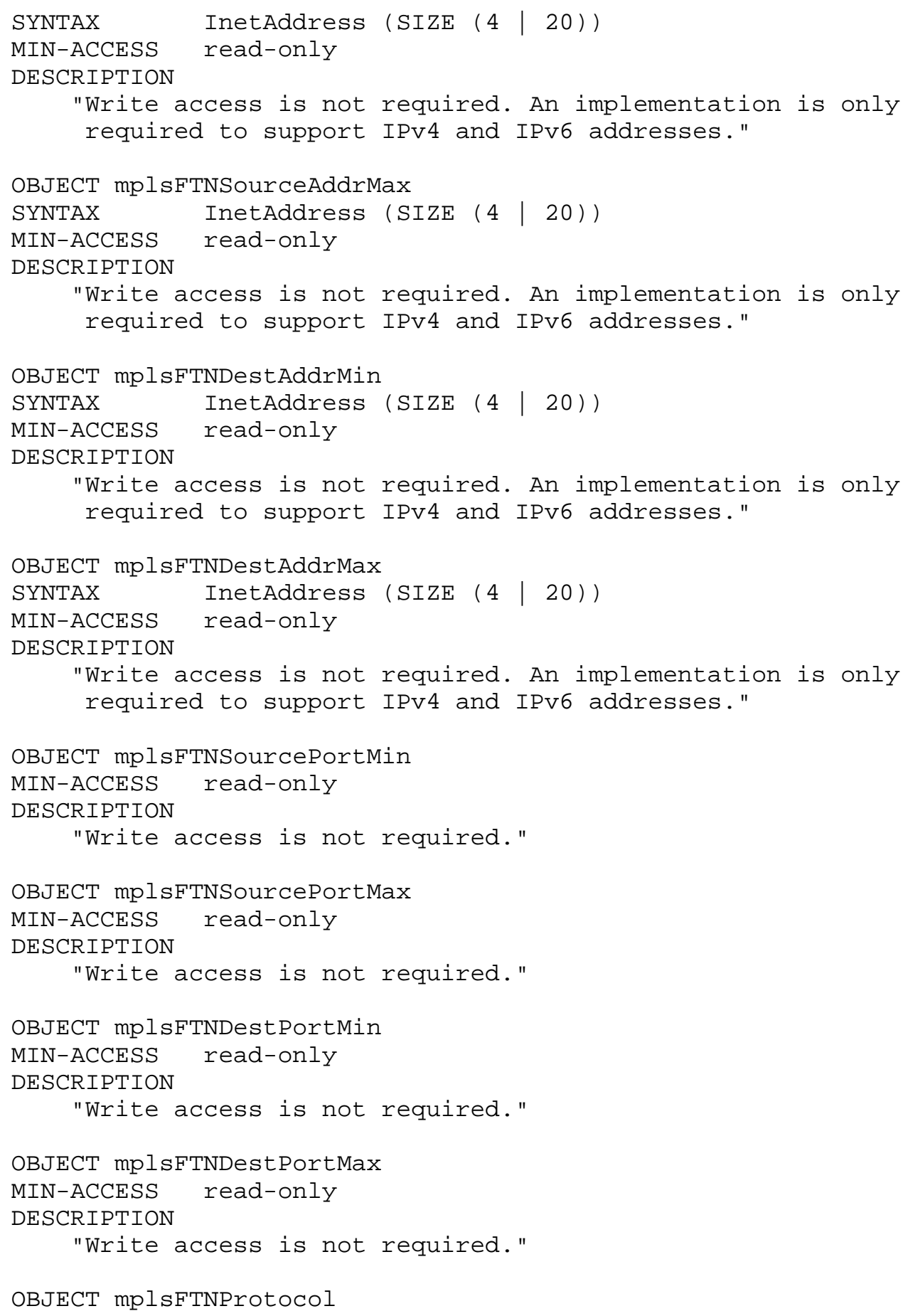




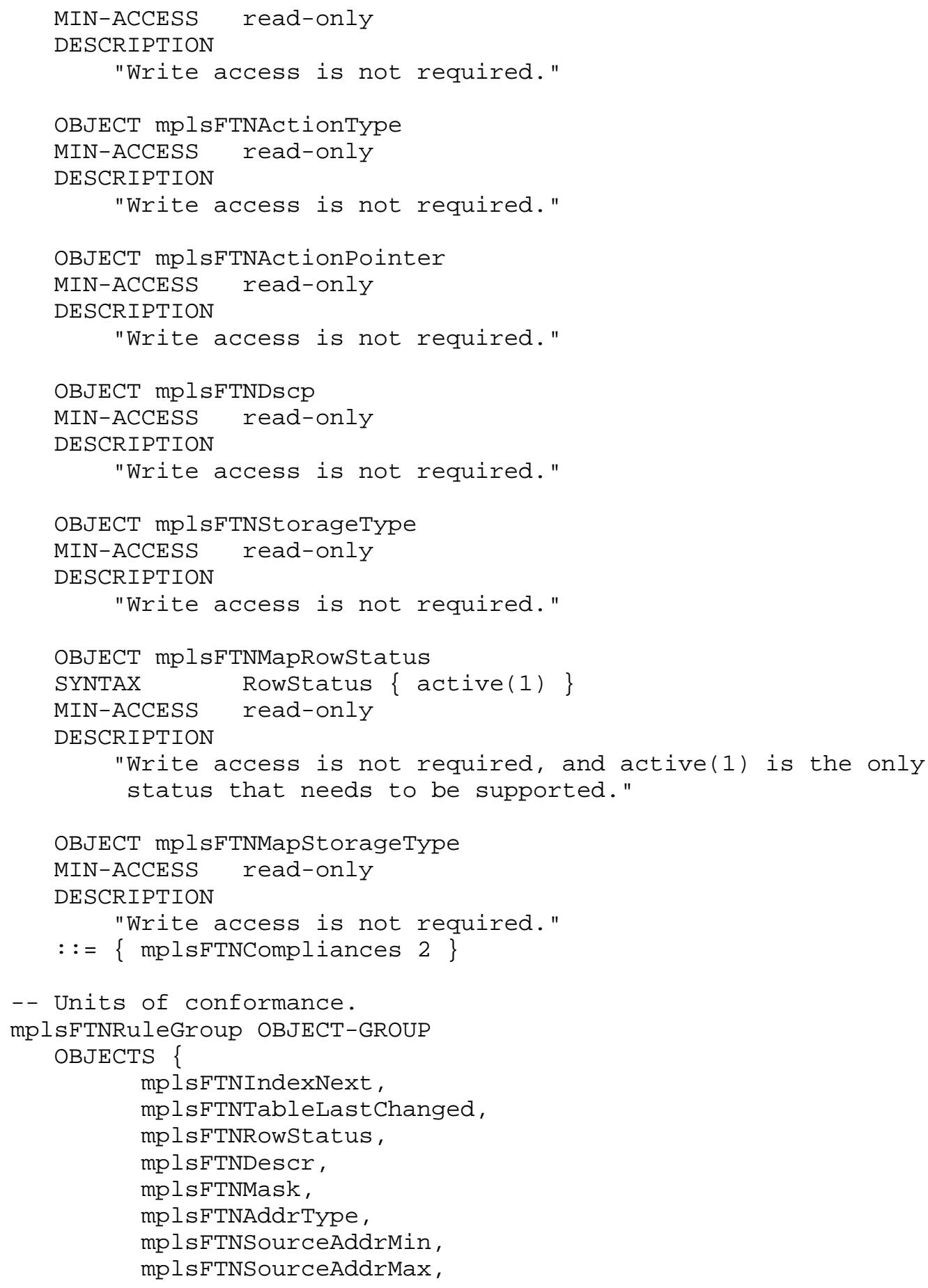




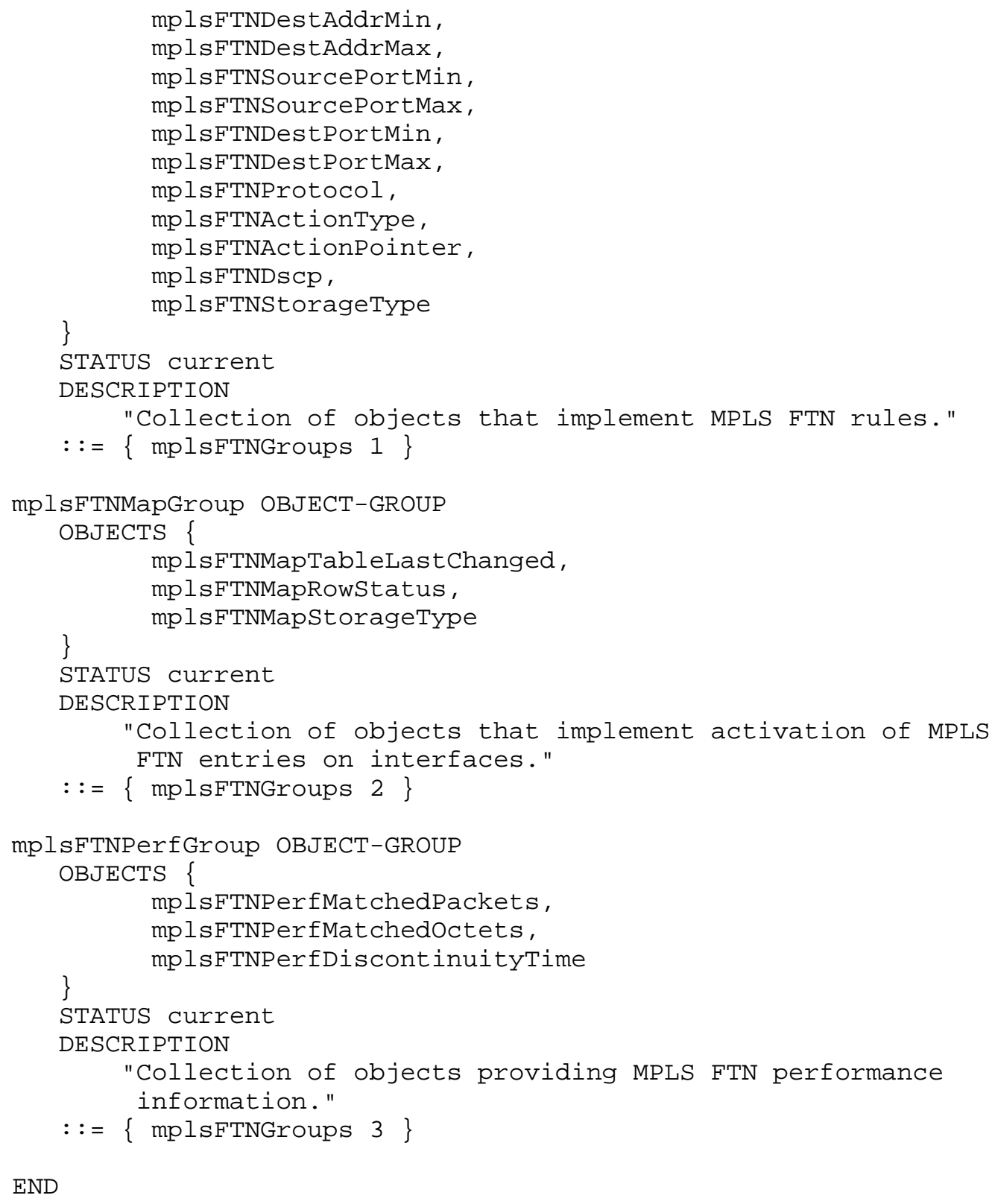

END 


\section{Security Considerations}

This MIB module can be used to configure LSRs to redirect non-MPLS traffic into an MPLS cloud. As such, improper manipulation of the objects represented in this MIB module may result in traffic being redirected to unintended destinations, potentially resulting in denial of service to end-users.

There are a number of management objects defined in this MIB module with a MAX-ACCESS clause of read-write and/or read-create. Such objects may be considered sensitive or vulnerable in some network environments. The support for SET operations in a non-secure environment without proper protection can have a negative effect on network operations. These are the tables and objects and their sensitivity/vulnerability:

- mplsfTnTable and mplsfTnMaptable can be used to create packet matching rules for classifying IPv4 or IPv6 traffic and redirecting matched packets into the MPLS cloud. Modifying objects in these tables can result in the misdirection of traffic and potential denial of service to end-users. It may also result in traffic which was intended to be redirected into the MPLS cloud being routed through the IP network instead, potentially resulting in degradation of service quality or outright denial of service.

Some of the readable objects in this MIB module (i.e., objects with a MAX-ACCESS other than not-accessible) may be considered sensitive or vulnerable in some network environments. It is thus important to control even GET and/or NOTIFY access to these objects and possibly to even encrypt the values of these objects when sending them over the network via SNMP. These are the tables and objects and their sensitivity/vulnerability:

- mplsfTNPerfTable provides counters for monitoring the performance of packet classification rules defined in mplsFTNTable and mplsfTNMapTable. Unauthorized read access to objects in these tables may be used to gain traffic flow information.

SNMP versions prior to SNMPv3 did not include adequate security. Even if the network itself is secure (for example by using IPSec), even then, there is no control as to who on the secure network is allowed to access and GET/SET (read/change/create/delete) the objects in this MIB module.

It is RECOMMENDED that implementers consider the security features as provided by the SNMPv3 framework (see [RFC3410], section 8), including full support for the SNMPv3 cryptographic mechanisms (for authentication and privacy). 
Further, deployment of SNMP versions prior to SNMPv3 is NOT RECOMMENDED. Instead, it is RECOMMENDED that SNMPv3 be deployed and cryptographic security be enabled. It is then a customer/operator responsibility to ensure that the SNMP entity giving access to an instance of this MIB module is properly configured to give access to the objects to only those principals (users) that have legitimate rights to indeed GET or SET (change/create/delete) them.

\section{IANA Considerations}

As described in [MPLSMGMT] and as requested in [RFC3811], MPLS related standards-track MIB modules should be rooted under the mplsstdMIB subtree. New assignments can only be made by a standards action as specified in [RFC2434].

\subsection{IANA Considerations for MPLS-FTN-STD-MIB}

The IANA has assigned mplsStdMIB 8 to the MPLS-FTN-STD-MIB module specified in this document.

\section{References}

12.1. Normative References

[RFC2119] Bradner, S., "Key Words for use in RFCs to Indicate Requirement Levels", BCP 14, RFC 2119, March 1997.

[RFC2578] McCloghrie, K., Perkins, D., and J. Schoenwaelder, "Structure of Management Information Version 2 (SMIv2)", STD 58, RFC 2578, April 1999.

[RFC2579] MCCloghrie, K., Perkins, D., and J. Schoenwaelder, "Textual Conventions for SMIv2", STD 58, RFC 2579, April 1999.

[RFC2580] MCCloghrie, K., Perkins, D., and J. Schoenwaelder, "Conformance Statements for SMIv2", STD 58, RFC 2580, April 1999.

[RFC2863] MCCloghrie, K. and F. Kastenholz, "The Interfaces Group MIB", RFC 2863, June 2000 .

[RFC3031] Rosen, E., Viswanathan, A., and R. Callon, "Multiprotocol Label Switching Architecture", RFC 3031, January 2001.

[RFC3289] Baker, F., Chan, K., and A. Smith, "Management Information Base for the Differentiated Services Architecture", RFC 3289, May 2002 . 
[RFC3291] Daniele, M., Haberman, B., Routhier, S., and J. Schoenwaelder, "Textual Conventions for Internet Network Addresses", RFC 3291, May 2002.

[RFC3411] Harrington, D., Presuhn, R., and B. Wijnen, "An Architecture for Describing Simple Network Management Protocol (SNMP) Management Frameworks", STD 62, RFC 3411, December 2002 .

[RFC3813] Srinivasan, C., Viswanathan, A., and T. Nadeau, "Multiprotocol Label Switching (MPLS) Label Switching Router (LSR) Management Information Base (MIB)", RFC 3813, June 2004 .

[RFC3811] Nadeau, T., and J. Cucchiara, J., Editors, "Definition of Textual Conventions (TCs) for Multi-Protocol Label Switching (MPLS) Management", RFC 3811, June 2004.

[RFC3812] Srinivasan, C., Viswanathan, A., and T. Nadeau, "Multiprotocol Label Switching (MPLS) Traffic Engineering (TE) Management Information Base (MIB)", RFC 3812, June 2004 .

12.2. Informative References

[MPLSMGMT] Nadeau, T., Srinivasan, C., and A. Farrel, "Multiprotocol Label Switching (MPLS) Management Overview", Work in Progress, September 2003.

[RFC791] Postel, J., "Internet Protocol", STD 5, RFC 791, September 1981 .

[RFC1519] Fuller, V., Li, T., Yu, J., and K. Varadhan, "Classless Inter-Domain Routing (CIDR) : an Address Assignment and Aggregation Strategy", RFC 1519, September 1993.

[RFC2026] Bradner, S., "The Internet Standards Process -- Revision 3", BCP 9, RFC 2026, October 1996.

[RFC2434] Narten, T. and H. Alvestrand, "Guidelines for Writing an IANA Considerations section in RFCs", BCP 26, RFC 2434, October 1998 .

[RFC2460] Deering, S. and R. Hinden, "Internet Protocol, Version 6 (IPv6) Specification", RFC 2460, December 1998. 
[RFC2474] Nichols, K., Blake, S., Baker, F., and D. Black, "Definition of the Differentiated Services Field (DS Field) in the IPv4 and IPv6 Headers", RFC 2474, December 1998 .

[RFC3410] Case, J., Mundy, R., Partain, D., and B. Stewart, "Introduction and Applicability Statements for InternetStandard Management Framework", RFC 3410, December 2002.

\title{
13. Acknowledgements
}

\begin{abstract}
We would particularly like to thank Bert Wijnen for the substantial time and effort he spent in helping us improve this document. We would also like to thank David Perkins, Joan Cucchiara, Mike Piecuch, and Adrien Grise for their insightful comments and additions to this document.
\end{abstract}

14. Authors' Addresses

Thomas D. Nadeau Cisco Systems, Inc. 300 Apollo Drive Chelmsford, MA 01824

Phone: +1-978-244-3051

EMail: tnadeau@cisco.com

Cheenu Srinivasan

Bloomberg L.P.

499 Park Avenue

New York, NY 10022

Phone: +1-212-893-3682

EMail: cheenulbloomberg. net

Arun Viswanathan

Force10 Networks, Inc. 1440 McCarthy Blvd Milpitas, CA 95035

Phone: +1-408-571-3516

EMail: arunveforcelonetworks.com 
15. Full Copyright statement

Copyright (C) The Internet society (2004). This document is subject to the rights, licenses and restrictions contained in BCP 78, and except as set forth therein, the authors retain all their rights.

This document and the information contained herein are provided on an "AS IS" basis and THE CONTRIBUTOR, THE ORGANIZATION HE/SHE REPRESENTS OR IS SPONSORED BY (IF ANY), THE INTERNET SOCIETY AND THE INTERNET ENGINEERING TASK FORCE DISCLAIM ALL WARRANTIES, EXPRESS OR IMPLIED, INCLUDING BUT NOT LIMITED TO ANY WARRANTY THAT THE USE OF THE INFORMATION HEREIN WILL NOT INERINGE ANY RIGHTS OR ANY IMPLIED WARRANTIES OF MERCHANTABILITY OR FITNESS FOR A PARTICULAR PURPOSE.

Intellectual Property

The IETF takes no position regarding the validity or scope of any Intellectual property Rights or other rights that might be claimed to pertain to the implementation or use of the technology described in this document or the extent to which any license under such rights might or might not be available; nor does it represent that it has made any independent effort to identify any such rights. Information on the procedures with respect to rights in RFC documents can be found in BCP 78 and BCP 79 .

Copies of IPR disclosures made to the IETF Secretariat and any assurances of licenses to be made available, or the result of an attempt made to obtain a general license or permission for the use of such proprietary rights by implementers or users of this specification can be obtained from the IETF on-line IPR repository at http://www.ietf.org/ipr.

The IETF invites any interested party to bring to its attention any copyrights, patents or patent applications, or other proprietary rights that may cover technology that may be required to implement this standard. Please address the information to the IETF at ietf-ipreietf.org.

Acknowledgement

Funding for the RFC Editor function is currently provided by the Internet Society. 